\title{
Transport properties in resonant tunneling heterostructures
}

\author{
Carlo Presillat \\ Dipartimento di Fisica, Universita di Roma "La Sapienza," \\ Piazzale A. Moro 2, 00185 Roma, Italy \\ Johannes Sjöstrand \\ Centre de Mathématique, Ecole Polytechnique, \\ F-91128 Palaiseau Cedex, France and U.A. 169, C.N.R.S. \\ (cond-mat/9607088 to be published in J. Math. Phys. 37/10 (1996))
}

\begin{abstract}
We use an adiabatic approximation in terms of instantaneous resonances to study the steadystate and time-dependent transport properties of interacting electrons in biased resonant tunneling heterostructures. This approach leads, in a natural way, to a transport model of large applicability consisting of reservoirs coupled to regions where the system is described by a nonlinear Schrödinger equation. From the mathematical point of view, this work is non-rigorous but may offer some fresh and interesting problems involving semiclassical approximation, adiabatic theory, non-linear Schrödinger equations and dynamical systems.

05.45.+b, 03.65.-w, 73.40.Gk
\end{abstract}

\section{INTRODUCTION}

Man-tailored semiconductor heterostructures [1] offer, for the first time, the possibility to test quantum mechanics at a mesoscopic level [2]. The scenario of systems which can be investigated is so rich that the art of their realization deserves the name of quantum design.

In the simplest case, a quantum designer can grow sandwiches of different semiconductor alloys by choosing the number of atomic layers for each kind of alloy. In the resulting heterostructure, the conduction band profile along the growth direction forms steps whose height can be continuously varied by a proper choice of the alloy composition. Typical widths and heights are of the order of tens of $\AA$ and tenths of eV, respectively.

At low-temperature, the mean free path of carriers for scattering from crystal impurities is of the order of $10^{4} \AA$ and for heterostructures smaller than this size the electric transport along the growth direction is a phase coherent quantum scattering from the conduction band discontinuities [3]. Due to the translational invariance in the plane orthogonal to the growth direction, the problem is one-dimensional. Moreover, the carriers are described by an effective mass which accounts for the microscopic scattering with the periodic crystal sites and their wave function is an envelope wave function $\llbracket$.

In a homogeneous neutral conductor, the electron-electron interaction can be taken into account by a renormalization of the carrier effective masses [-5] and one deals with a transport problem like in a noninteracting case. In a heterostructure, even as simple as that described above, the breaking of translational invariance in the transport direction allows the electric neutrality to be locally violated. The corresponding interaction potential, obtained, at Hartree level, by solving a proper Poisson equation, can strongly modify the transport properties. The example of a double barrier heterostructure with the exterior regions doped with donors is illuminating [6]. Due to tunneling, electrons populate the resonance(s) created by the double barrier and the region between the barriers becomes negatively charged. This generates an electric potential which decreases the tunneling probability of electrons in the double

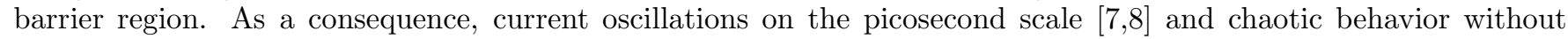
classical counterpart [9] have been predicted in a ballistic configuration in which electrons are injected at some chosen energy.

Experiments with ballistic electrons are difficult and measurements became available only recently [10]. Technologically simpler is the case of biased heterostructures where transport is due to the presence of reservoirs at thermal equilibrium with different chemical potentials. Manifestations of the electron-electron interaction are known also in this configuration. For example, hysteresis in the current-voltage characteristics of double barrier heterostructures have been observed [11] and recognized as a consequence of the accumulation of electrons in the resonance [11 15]. In this case, however, one has the theoretical problem of attaching reservoirs at thermal equilibrium to a piece of conductor where quantum coherent transport takes place.

In the recent paper [16] we proposed an approach to this problem based on a mathematical method earlier applied in the framework of ballistic transport [17]. We showed that for heterostructures with a single resonance our approach allows one $i$ ) to obtain steady-state voltage-current characteristics having hysteresis or not in agreement with the 
experimental results [18] and ii) to predict time-dependent properties analogous to those studied in optically bistable systems [19]. Here, we develop the general mathematical scheme of this approach and discuss the case with several resonances where multistability phenomena can take place as in superlattices 20.21.

For simplicity, consider the one-dimensional double barrier heterostructure discussed above. The idea is that due to the presence of resonances the corresponding Schrödinger problem can be divided in two parts: a Schrödinger equation for the barrier region and one for the exterior space, the two being weakly coupled by tunneling. This decomposition corresponds to the schematization of the transport process as a coherent process fed by reservoirs. In the exterior space (reservoirs) homogeneous and neutral, the electron-electron interaction is neglected and thermal equilibrium is taken into account by considering a continuous set of energy eigenstates distributed according to the Fermi statistics. In the barrier region (coherent conductor), the Coulomb interaction is included in a self-consistent potential obtained by solving the Poisson equation associated to the local charge density. Under the assumption that the barriers are wide enough, the corresponding nonlinear Schrödinger problem is discussed in two steps. In the first step we eliminate the potential well between the two barriers, by artificially increasing the potential there, and we solve the Schrödinger equation asymptotically for the new potential by means of WKB-expansions. The resulting solution is then very small near the (filled) potential well, so we get only a small error in the Schrödinger equation when we go back to the true potential. In the second step we correct for this small error by adding a wave function concentrated near the potential well. Assuming a priori that the charge in the well changes slowly with time, the correcting wave function can be expected to be large only at energies close to the resonances, and be well approximated by some linear combination of the resonant states.

In most of the paper we discuss the case in which only one resonance participates. The validity of this one-mode approximation has been tested numerically with excellent results in the ballistic configuation of [17]. Here, the coefficient of the one-mode approximation obeys an ordinary differential equation with respect to time in the infinite dimensional space of square integrable functions of energy. We study the stationary points of the corresponding vector field and their nature, whether they are attractive or not, and arrive at quite neat answers. For solutions of the dynamical system which have existed as bounded solutions for a long time and in a suitable asymptotic limit (of wide barriers) we derive a simplified scalar differential equation for the evolution of the sheet density of electrons trapped in well which gives good global understanding of the more complete dynamical system. Using these results, we are able to discuss the phenomenon of hysteresis and we support and illustrate the discussion with several numerical results. The discussion includes the evolution of solutions away from fixed points which necessarily appears when there is hysteresis. We also discuss the case of several resonances, and get analogous results.

From the mathematical point of view, the present paper could be a starting point for rigorous work on some fresh problems, involving semiclassical analysis, adiabatic theory, non-linear Schrödinger equations and dynamical systems. A strong motivation for such an enterprise is the fact that the theory of electric transport in semiconductor devices offers many problems similar to that one we illustrate here [22].

The plan of the paper is as follows. In Section II we define the model. In Section III we review the WKB expansion for slowly varying potentials. In Sections IV and $\mathrm{V}$ we determine the driving term and the ground resonant state, respectively, within the WKB approximation. The central equation of our paper is derived in Section VI and the general properties of the associated fixed points and linearizations are discussed in Section VII. In Section VIII we introduce an approximation valid in the limit of small resonance width and discuss the corresponding fixed point solutions and linearizations. In section $\mathrm{IX}$ we obtain a simplified differential equation describing the dynamics of the electron density in the well. A qualitative discussion of the hysteresis phenomenon in comparison with numerical results is given in Section $\mathrm{X}$. In Section XI we finally consider the case with several resonances.

\section{DEFINITION OF THE MODEL}

Let us consider a heterostructure whose conduction band profile consists of two barriers of height $V_{0}$ located in $[a, b]$ and $[c, d]$

$$
V_{\mathrm{cb}}(x)=\left\{\begin{array}{lr}
0 & x<a \\
V_{0} & a<x<b \\
0 & b<x<c \\
V_{0} & c<x<d \\
0 & x>d
\end{array}\right.
$$

with $a<b<c<d$ along the growth direction $x$. We wish to evaluate the transport properties of this device when a bias energy $\Delta V$ is applied between the emitter $(x<a)$ and collector $(x>d)$ regions uniformly doped. Due to doping, the band of conduction electrons formed in the emitter and collector regions is characterized by a Fermi 
energy $E_{F}=\left(3 \pi^{2} n_{D}\right)^{2 / 3}$, where $n_{D}$ is the net donor concentration. We will use everywhere effective atomic units $\hbar=2 m^{*}=1$ and $e^{2} / \varepsilon=2 a_{B}^{-1}$, where $m^{*}$ is the electron effective mass and $\varepsilon$ the dielectric constant. In these units, every physical quantity is expressed in terms of the effective Bohr radius $a_{B}=\hbar^{2} \varepsilon /\left(m^{*} e^{2}\right)$. Assuming an ideal heterostructure homogeneous in the plane $y z$ parallel to the junctions (and orthogonal to the growth direction $x$ ), the single-electron momenta $k_{y}$ and $k_{z}$ are conserved quantities. As a consequence, the single-electron wavefunction at energy $E+E_{\|}$, where $E_{\|}=k_{y}^{2}+k_{z}^{2}$, can be factorized as $\phi(x, t, E) \chi\left(y, z, t, E_{\|}\right)$with

$$
\chi\left(y, z, t, E_{\|}\right)=\frac{1}{\sqrt{A}} e^{i\left(k_{y} y+k_{z} z\right)} e^{-i E_{\|} t} .
$$

We will assume periodic boundary conditions in a two-dimensional region $A$ so that the momenta $k_{y}$ and $k_{z}$ are quantized as in a real device having finite lateral area of size $A$. The time dependent Schrödinger equation for the single-electron wavefunction at energy $E$ along the $x$ direction is

$$
\left[-i \partial_{t}-\partial_{x}^{2}+V_{\mathrm{cb}}(x)+U(\phi, x)\right] \phi(x, t, E)=0,
$$

where $U(\phi, x)$ takes into account the applied bias and, at Hartree level, the electron-electron interaction. Assuming ideal metallic behavior in the emitter and collector regions, i.e., neglecting the formation of accumulation and depletion layers, $U(\phi, x)$ can be obtained as solution of the Poisson equation

$$
\partial_{x}^{2} U(\phi, x)=-8 \pi a_{B}^{-1} \rho(\phi)
$$

with Dirichlet boundary conditions $U(\phi, a)=0$ and $U(\phi, d)=-\Delta V$. The density $\rho$ takes into account all the electrons in the occupied energy states and depends only on the wavefunction component $\phi$. Indeed, if the emitter and collector regions are at thermal equilibrium with temperature $T$ we have

$$
\begin{aligned}
\rho & =2 \int_{0}^{\infty} d E \sum_{E_{\|}}\left|\phi(x, t, E) \chi\left(y, z, t, E_{\|}\right)\right|^{2}\left(1+e^{\frac{E+E_{\|}-E_{F}}{k_{B} T}}\right)^{-1} \\
& =\int d E g(E)|\phi(x, t, E)|^{2}
\end{aligned}
$$

where the factor 2 takes into account the spin degeneracy. Energies are measured from the bottom of the emitter conduction band and the lower integration bound $E=0$ in the first line of (2.5) stems from the fact that for $E_{F} \ll \Delta V$, as we will assume, only electrons from the emitter conduction band can penetrate the region $[a, d]$ where the electron density is of interest. In the second line of (2.5) this lower bound is absorbed in the definition of $g(E)$ by a Heaviside function $\theta(E)$. The function $g(E)$ can be explicitly evaluated by approximating the sum over the parallel degrees of freedom with an integral

$$
\begin{aligned}
g(E) & =\theta(E) 2 \int_{0}^{\infty} d E_{\|} \frac{A}{4 \pi}\left|\frac{1}{\sqrt{A}}\right|^{2}\left(1+e^{\frac{E+E_{\|}-E_{F}}{k_{B} T}}\right)^{-1} \\
& =\theta(E) \frac{1}{2 \pi}\left[k_{B} T \ln \left(1+e^{\frac{E-E_{F}}{k_{B} T}}\right)+E_{F}-E\right] .
\end{aligned}
$$

Note that the chemical potential at temperature $T$ in the Fermi function has been approximated with its value at $T=0$, i.e., the Fermi energy determined by the net donor concentration.

In general, the solution of (2.4) can not be handled analytically. We will suppose that, due to the accumulation of electrons in the well with sheet density

$$
s(\phi)=\int d E g(E) \int_{(a+b) / 2}^{(c+d) / 2} d x|\phi(x, t, E)|^{2},
$$

ideal metallic behavior in the well $[b, c]$ and ideal insulating behavior in the barriers $[a, b]$ and $[c, d]$ hold. This is equivalent to approximate (2.4) with

$$
\partial_{x}^{2} U(\phi, x)=-8 \pi a_{B}^{-1} s(\phi)[B \delta(x-b)+C \delta(x-c)], \quad B+C=1
$$

and the condition that $\partial_{x} U(\phi, x)=0$ for $b<x<c$. In this case $U(\phi, x)$ becomes a piece-wise linear function of $x$ with $\partial_{x} U(\phi, x)$ having jump discontinuouities at $x=b$ and $x=c$. The total potential $V_{\mathrm{cb}}+U$ in (2.3) is better rewritten as $V+W$ where 


$$
V(x)=\left\{\begin{array}{lr}
0 & x<a \\
V_{0}-\Delta V(x-a) / \ell & a<x<b \\
-\Delta V(b-a) / \ell & b<x<c \\
V_{0}-\Delta V(b-a+x-c) / \ell & c<x<d \\
-\Delta V & x>d
\end{array}\right.
$$

gives the band profile modified by the external bias and

$$
W(s, x)=8 \pi a_{B}^{-1} s(\phi)\left\{\begin{array}{lr}
0 & x<a \\
(x-a)(d-c) / \ell & a<x<b \\
(b-a)(d-c) / \ell & b<x<c \\
(b-a)(d-x) / \ell & c<x<d \\
0 & x>d
\end{array}\right.
$$

depends on the wavefunction $\phi$ through the sheet density of electrons in the well $s(\phi)$. Here $\ell=b-a+d-c$. The potentials $V(x)$ and $W(s, x)$ are shown in Fig. 1.

We will try to solve the nonlinear partial differential equation

$$
\left[-i \partial_{t}-\partial_{x}^{2}+V(x)+W(s, x)\right] \phi(x, t, E)=0,
$$

where $s(\phi)$ is given by (2.7), in two steps. Let $V_{\text {fill }}(x)=V(x)+V_{0} 1_{[b, c]}(x)$ be the potential obtained by filling the well $[b, c]$. Here $1_{[b, c]}(x)$ is the characteristic function of the interval $[b, c]$. First we solve

$$
\left[-i \partial_{t}-\partial_{x}^{2}+V_{\text {fill }}(x)+W(s, x)\right] \tilde{\mu}(x, t, E)=0
$$

and then we look for $\phi$ in the form $\phi=\tilde{\mu}+\tilde{\nu}$ where $\tilde{\nu}$ should solve

$$
\left[-i \partial_{t}-\partial_{x}^{2}+V(x)+W(s, x)\right] \tilde{\nu}(x, t, E)=V_{0} 1_{[b, c]}(x) \tilde{\mu}(x, t, E) .
$$

The wave function $\tilde{\mu}$ describes an electron at energy $E$ which is delocalized in the emitter and collector regions and has an exponentially small probability to be found in the forbidden region $[a, d]$. The wave function $\tilde{\nu}$ describes the localization, driven by $\tilde{\mu}$, of the same electron in the well $[b, c]$. The wave function $\phi$ of the original problem (2.11) can be approximated by $\tilde{\nu}$ or $\tilde{\mu}$ inside or outside the two barriers, respectively, with an error which is exponentially small in the limit of wide barriers [17].

To evaluate $\tilde{\mu}$ we will use a WKB approximation in the forbidden region $[a, d]$. Equation (2.13) will be treated with a one mode approximation in which $\tilde{\nu}$ is assumed proportional to a resonant state corresponding to the potential $V+W$. To evaluate this resonant state and the corresponding resonance, we will again use a WKB approximation. In both cases, the justification of using a WKB approximation stems from the fact that $V_{\text {fill }}+W$ and $V+W$ are slowly varying potentials in the barriers regions if $b-a$ and $d-c$ are large while $\Delta V$ and $s$ remain bounded.

\section{WKB EXPANSION FOR SLOWLY VARYING POTENTIALS}

Let $\mathcal{U}=\mathcal{U}_{h}(x)$ be a real valued potential on some interval, with $\partial_{x} \mathcal{U}=\mathcal{O}(|h|)$ and $\partial_{x}^{2} \mathcal{U}=\mathcal{O}\left(h^{2}\right)$, where $|h| \ll 1$ is a parameter. Let $\mathcal{E}$ be a real energy and assume that $\mathcal{U}_{h}(x)-\mathcal{E}$ is bounded from above and from below by some strictly positive constants that are independent of $h$. This means that we are in the classically forbidden region. Then

$$
\begin{aligned}
& {\left[-\partial_{x}^{2}+\mathcal{U}-\mathcal{E}\right](\mathcal{U}-\mathcal{E})^{-1 / 4} e^{-\int{ }^{x} d x^{\prime}(\mathcal{U}-\mathcal{E})^{1 / 2}}} \\
& =\left[-\frac{5}{16}(\mathcal{U}-\mathcal{E})^{-9 / 4}\left(\partial_{x} \mathcal{U}\right)^{2}+\frac{1}{4}(\mathcal{U}-\mathcal{E})^{-5 / 4} \partial_{x}^{2} \mathcal{U}\right] e^{-\int^{x} d x^{\prime}(\mathcal{U}-\mathcal{E})^{1 / 2}} \\
& =e^{-\int^{x} d x^{\prime}(\mathcal{U}-\mathcal{E})^{1 / 2}} \mathcal{O}\left(h^{2}\right),
\end{aligned}
$$

and therefore

$$
(\mathcal{U}-\mathcal{E})^{-1 / 4} e^{-\int^{x} d x^{\prime}(\mathcal{U}-\mathcal{E})^{1 / 2}}
$$

is a good approximation to a corresponding exact eigenfunction, even over intervals of length $\mathcal{O}\left(|h|^{-1}\right)$.

In the following sections, we will apply the above approximation in the barrier regions $[a, b]$ and $[c, d]$ with $h$ equal to the $x$ derivative of $V+W$ in these intervals. 


\section{THE DRIVING TERM}

Equation (2.12) can be solved by evaluating the instantaneous eigenstates of the potential $V_{\text {fill }}+W$. We put $\tilde{\mu}(x, t, E)=\exp (-i E t) \mu(x, t, E)$ and suppose that $\Delta V$ and $s$ are slowly varying functions of time so that also $\mu(x, t, E)$ is slowly varying in time. Thus in the equation

$$
\left[-i \partial_{t}-\partial_{x}^{2}+V_{\text {fill }}(x)+W(s, x)-E\right] \mu(x, t, E)=0,
$$

we make a very small error if we neglect the term $-i \partial_{t} \mu$, as we shall do in the following. In the emitter region $x<a$, we take $\mu(x, t, E)$ as the sum of a left and a right-going plane wave at energy $E$

$$
\mu(x, t, E)=\frac{1}{\sqrt{4 \pi \sqrt{E}}}\left(e^{i \sqrt{E}(x-a)}+r(E) e^{-i \sqrt{E}(x-a)}\right)
$$

where $r(E)$ is a reflection amplitude to be computed. Note that the normalization factor in (4.2) is chosen in order to have $\int d x \overline{\mu(x, t, E)} \mu\left(x, t, E^{\prime}\right)=\delta\left(E-E^{\prime}\right)$ in agreement with the expression of the electron density (2.5) in terms of an integral over the energy $E$. We propagate the expression (4.2) to the adjacent regions by requiring $\mu$ to be of class $C^{1}$ and applying the WKB approximation described in Section III. In the interval $[a, b]$ the potential is $V_{\text {fill }}+W=V_{0}+\alpha(x-a)$, where

$$
\alpha=\frac{8 \pi a_{B}^{-1} s(d-c)-\Delta V}{b-a+d-c}
$$

plays the role of the small parameter $h$ of Section III. For $a<x<b$ we can then use the WKB approximation

$$
\mu(x, t, E)=\frac{1}{\sqrt{4 \pi \sqrt{E}}} \frac{\left(V_{0}-E\right)^{1 / 4}}{\left(V_{0}+\alpha(x-a)-E\right)^{1 / 4}} t(E) e^{-\int_{a}^{x} d x^{\prime}\left(V_{0}+\alpha\left(x^{\prime}-a\right)-E\right)^{1 / 2}}
$$

where $t(E)$ is a transmission amplitude to be determined with $r(E)$ from the $C^{1}$ condition at $x=a$

$$
\begin{aligned}
1+r(E) & =t(E) \\
i \sqrt{E}-i \sqrt{E} r(E) & =t(E)\left[\left(V_{0}-E\right)^{1 / 2}-\frac{1}{4}\left(V_{0}-E\right)^{-5 / 4} \alpha\right] .
\end{aligned}
$$

Neglecting the last term in the bracket, which is $\mathcal{O}(|\alpha|)$, we get

$$
\begin{aligned}
r(E) & =\frac{1+i\left(V_{0} / E-1\right)^{1 / 2}}{1-i\left(V_{0} / E-1\right)^{1 / 2}} \\
t(E) & =\frac{2}{1-i\left(V_{0} / E-1\right)^{1 / 2}} .
\end{aligned}
$$

Note that the neglected term would give correction factors $1+\mathcal{O}(|\alpha|)$ to $r(E)$ and $t(E)$.

At $x=b$ we can set up a similar transition problem but here $V_{\text {fill }}+W$ is continuous and the corresponding transmission amplitude is $1+\mathcal{O}(|\alpha|)$. Neglecting again a factor $1+\mathcal{O}(|\alpha|)$, for $b<x<c$ we get

$$
\mu(x, t, E)=\mu_{0}(t, E) e^{-\left(V_{0}+\alpha(b-a)-E\right)^{1 / 2}(x-b)}
$$

where

$$
\mu_{0}(t, E)=\frac{1}{\sqrt{4 \pi \sqrt{E}}} \frac{\left(V_{0}-E\right)^{1 / 4}}{\left(V_{0}+\alpha(x-a)-E\right)^{1 / 4}} \frac{2 e^{\left[\left(V_{0}-E\right)^{3 / 2}-\left(V_{0}+\alpha(b-a)-E\right)^{3 / 2}\right] 2 / 3 \alpha}}{1+i\left(V_{0} / E-1\right)^{1 / 2}} .
$$

Only this expression of $\mu$ in the region $[b, c]$ will be used in the following as driving term of Eq. (2.13). 


\section{RESONANCE AND RESONANT STATE}

In this section we will obtain a WKB approximate expression for the ground state resonance $\lambda(s)=E_{R}(s)-i \Gamma(s) / 2$ and the corresponding resonant state $e(s, x)$ for the potential $V+W$. We will assume that $c-b$ is bounded from below and from above by positive constants, while $b-a$ and $d-c$ are large enough.

To start with, we recall the construction of the ground state eigenvalue $E_{0}^{w}$ of the potential $V_{w}(x)=$ $V_{0}\left[1_{]-\infty, b]}(x)+1_{[c,+\infty[}(x)\right]$ which coincides, up to the constant shift

$$
\Delta E=\frac{8 \pi a_{B}^{-1} s(b-a)(d-c)-\Delta V(b-a)}{b-a+d-c},
$$

with the potential $V+W$ in the well region $[b, c]$. The corresponding ground eigenstate is

$$
e_{0}^{w}(x)=C_{0}^{w}\left\{\begin{array}{lr}
\cos \left(\sqrt{E_{0}^{w}}(c-b) / 2\right) e^{-\left(V_{0}-E_{0}^{w}\right)^{1 / 2}(b-x)} & x<b \\
\cos \left(\sqrt{E_{0}^{w}}(x-(b+c) / 2)\right) & b<x<c \\
\cos \left(\sqrt{E_{0}^{w}}(c-b) / 2\right) e^{-\left(V_{0}-E_{0}^{w}\right)^{1 / 2}(x-c)} & x>c
\end{array}\right.
$$

where $0<E_{0}^{w}<\min \left(V_{0}, \pi^{2} /(c-b)^{2}\right)$ is determined by the requirement that $e_{0}^{w}(x)$ is of class $C^{1}$

$$
\tan \left(\sqrt{E_{0}^{w}}(c-b) / 2\right)=\left(V_{0} / E_{0}^{w}-1\right)^{1 / 2},
$$

and the normalization constant is

$$
C_{0}^{w}=\left[\frac{E_{0}^{w}}{V_{0}\left(V_{0}-E_{0}^{w}\right)^{1 / 2}}+\frac{c-b}{2}+\frac{\left(V_{0}-E_{0}^{w}\right)^{1 / 2}}{V_{0}}\right]^{-1 / 2}
$$

where we used the identities $\cos ^{2} u=\left(1+\tan ^{2} u\right)^{-1},(\sin 2 u) / 2=\sin u \cos u=\tan u\left(1+\tan ^{2} u\right)^{-1}$. In the following we will assume that $E_{0}^{w}+\Delta E<V_{0}-\Delta V$.

Next we look at the ground state of the potential

$$
V_{b}(x)=\left\{\begin{array}{lr}
V_{0}+\alpha(a-b) & x<a \\
V_{0}+\alpha(x-b) & a<x<b \\
0 & b<x<c \\
V_{0}+\beta(x-c) & c<x<d \\
V_{0}+\beta(d-c) & x>d
\end{array}\right.
$$

which coincides, up to the constant shift $\Delta E$, with $V+W$ on the larger region $[a, d]$ which includes the barriers. In Eq. (5.5) $\alpha$ is given by (4.3) and

$$
\beta=\frac{-8 \pi a_{B}^{-1} s(b-a)-\Delta V}{b-a+d-c} .
$$

Note that the potential $V_{b}$ has been obtained by bending the barriers of $V_{w}$ in the intervals $[a, b]$ and $[c, d]$ proportionally to $\alpha$ and $\beta$, respectively. Let $E_{0}^{b}$ be the ground state of $V_{b}$ and $e_{0}^{b}(x)$ the corresponding eigenfunction. Since $|\alpha|$ and $|\beta|$ are small, from the same WKB considerations of Section III we have $E_{0}^{b}=E_{0}^{w}+\mathcal{O}(|\alpha|+|\beta|)$ and $e_{0}^{b}(x)=e_{0}^{w}(x)+\mathcal{O}(|\alpha|+|\beta|)$. To get the leading asymptotics of the resonance width, we need to determine the linear contribution to $\mathcal{O}(|\alpha|+|\beta|)$ in $E_{0}^{b}$. By differentiating the eigenvalue equation for the potential $V_{b}$, we have

$$
\begin{aligned}
& \left.\partial_{\alpha} E_{0}^{b}\right|_{\alpha=\beta=0}=\left.\int_{-\infty}^{+\infty} d x \overline{e_{0}^{b}(x)} \partial_{\alpha} V_{b}(x)\right|_{\alpha=\beta=0} e_{0}^{b}(x) \simeq \int_{-\infty}^{b} d x(x-b)\left|e_{0}^{w}(x)\right|^{2} \\
& \left.\partial_{\beta} E_{0}^{b}\right|_{\alpha=\beta=0}=\left.\int_{-\infty}^{+\infty} d x \overline{e_{0}^{b}(x)} \partial_{\beta} V_{b}(x)\right|_{\alpha=\beta=0} e_{0}^{b}(x) \simeq \int_{c}^{+\infty} d x(x-c)\left|e_{0}^{w}(x)\right|^{2}
\end{aligned}
$$

and using (5.2) we get 


$$
\begin{aligned}
\left.\partial_{\alpha} E_{0}^{b}\right|_{\alpha=\beta=0}=-\left.\partial_{\beta} E_{0}^{b}\right|_{\alpha=\beta=0} & =\left(C_{0}^{w}\right)^{2} \cos ^{2}\left(\sqrt{E_{0}^{w}}(c-b) / 2\right) \int_{-\infty}^{0} d x x e^{2\left(V_{0}-E_{0}^{w}\right)^{1 / 2} x} \\
& =-\frac{\left(C_{0}^{w}\right)^{2} E_{0}^{w}}{4 V_{0}\left(V_{0}-E_{0}^{w}\right)}
\end{aligned}
$$

Observing that $\alpha-\beta=8 \pi a_{B}^{-1} s$, we finally get

$$
E_{0}^{b}=E_{0}^{w}-8 \pi a_{B}^{-1} s \frac{\left(C_{0}^{w}\right)^{2} E_{0}^{w}}{4 V_{0}\left(V_{0}-E_{0}^{w}\right)}+\mathcal{O}\left(\alpha^{2}+\beta^{2}\right)
$$

The real part $E_{R}(s)$ of the shape resonance of $-\partial_{x}^{2}+V+W$ which is close to the ground state eigenvalue of $-\partial_{x}^{2}+V_{b}+\Delta E$ is very well approximated by the above calculated $E_{0}^{b}+\Delta E$ which can be rewritten as

$$
E_{R}(s)=E_{R}(0)+\eta s,
$$

where

$$
E_{R}(0)=E_{0}^{w}-\Delta V(b-a) / \ell
$$

and

$$
\eta=\frac{8 \pi a_{B}^{-1}(b-a)(d-c)}{b-a+d-c}-\frac{8 \pi a_{B}^{-1}\left(C_{0}^{w}\right)^{2} E_{0}^{w}}{4 V_{0}\left(V_{0}-E_{0}^{w}\right)} .
$$

Now we discuss the determination of the imaginary part $\Gamma(s)$ of the resonance. In the interval $[a, d]$ the ground state of $V_{b}$ is

$$
e_{0}^{b}(x)=C_{0}^{b} \begin{cases}\frac{\cos \left(\sqrt{E_{0}^{b}}(c-b) / 2\right)\left(V_{0}-E_{0}^{b}\right)^{1 / 4}}{\left(V_{0}+\alpha(x-b)-E_{0}^{b}\right)^{1 / 4}} e^{-\int_{x}^{b} d x^{\prime}\left(V_{0}+\alpha\left(x^{\prime}-b\right)-E_{0}^{b}\right)^{1 / 2}} & a<x<b \\ \cos \left(\sqrt{E_{0}^{b}}(x-(b+c) / 2)\right) & b<x<c \\ \frac{\cos \left(\sqrt{E_{0}^{b}}(c-b) / 2\right)\left(V_{0}-E_{0}^{b}\right)^{1 / 4}}{\left(V_{0}+\beta(x-c)-E_{0}^{b}\right)^{1 / 4}} e^{-\int_{c}^{x} d x^{\prime}\left(V_{0}+\beta\left(x^{\prime}-c\right)-E_{0}^{b}\right)^{1 / 2}} & c<x<d\end{cases}
$$

where $C_{0}^{b}=C_{0}^{w}+\mathcal{O}(|\alpha|+|\beta|)$. In the interval $[a, d]$, the resonant state $e(s, x)$ can be approximated by adding to (5.14) terms due to reflections at $x=a$ and $x=d$. For $x \lesssim d$ we try with

$$
\begin{aligned}
e(s, x)= & \frac{C_{0}^{w} \cos \left(\sqrt{E_{0}^{b}}(c-b) / 2\right)\left(V_{0}-E_{0}^{b}\right)^{1 / 4}}{\left(V_{0}+\beta(x-c)-E_{0}^{b}\right)^{1 / 4}} e^{-\int_{c}^{d} d x^{\prime}\left(V_{0}+\beta\left(x^{\prime}-c\right)-E_{0}^{b}\right)^{1 / 2}} \\
& \times\left(e^{-\left(V_{0}+\beta(d-c)-E_{0}^{b}\right)^{1 / 2}(x-d)}+r e^{\left(V_{0}+\beta(d-c)-E_{0}^{b}\right)^{1 / 2}(x-d)}\right),
\end{aligned}
$$

where we have also replaced the exponent with its linear approximation at $x=d$. For $x \gtrsim d$ we try the right-going plane wave

$$
e(s, x)=\frac{C_{0}^{w} \cos \left(\sqrt{E_{0}^{b}}(c-b) / 2\right)\left(V_{0}-E_{0}^{b}\right)^{1 / 4}}{\left(V_{0}+\beta(d-c)-E_{0}^{b}\right)^{1 / 4}} e^{-\int_{c}^{d} d x^{\prime}\left(V_{0}+\beta\left(x^{\prime}-c\right)-E_{0}^{b}\right)^{1 / 2}} t e^{i\left(E_{0}^{b}-\beta(d-c)\right)^{1 / 2}(x-d)} .
$$

The $C^{1}$ condition at $x=d$ gives, up to terms $\mathcal{O}(|\beta|)$,

$$
\begin{aligned}
1+r & =t \\
-\left(V_{0}+\beta(d-c)-E_{0}^{b}\right)^{1 / 2}+\left(V_{0}+\beta(d-c)-E_{0}^{b}\right)^{1 / 2} r & =i\left(E_{0}^{b}-\beta(d-c)\right)^{1 / 2} t
\end{aligned}
$$

which determines $r$ and $t$ so that for $x \gtrsim d$ we have

$$
\begin{aligned}
& e(s, x)=\frac{C_{0}^{w} \cos \left(\sqrt{E_{0}^{b}}(c-b) / 2\right)\left(V_{0}-E_{0}^{b}\right)^{1 / 4}}{\left(V_{0}+\beta(d-c)-E_{0}^{b}\right)^{1 / 4}} 2\left[1-i \frac{\left(E_{0}^{b}-\beta(d-c)\right)^{1 / 2}}{\left(V_{0}+\beta(d-c)-E_{0}^{b}\right)^{1 / 2}}\right]^{-1} \\
& \times \exp \left\{\frac{2}{3 \beta}\left[\left(V_{0}-E_{0}^{b}\right)^{3 / 2}-\left(V_{0}+\beta(d-c)-E_{0}^{b}\right)^{3 / 2}\right]+i\left(E_{0}^{b}-\beta(d-c)\right)^{1 / 2}(x-d)\right\} .
\end{aligned}
$$


In these calculations we have assumed that $E_{0}^{b}-\beta(d-c)>0, V_{0}+\beta(d-c)-E_{0}^{b}>0$. The first inequality is always fulfilled in experimentally relevant situations, while the second one, equivalent to $E_{R}(s)<V_{0}-\Delta V$ may be more critical and, possibly, one should replace (5.18) by a more complicated formula.

The same calculation can be repeated for $x=a$. For $x \gtrsim a$ we try with

$$
\begin{aligned}
e(s, x)= & \frac{C_{0}^{w} \cos \left(\sqrt{E_{0}^{b}}(c-b) / 2\right)\left(V_{0}-E_{0}^{b}\right)^{1 / 4}}{\left(V_{0}+\alpha(x-b)-E_{0}^{b}\right)^{1 / 4}} e^{-\int_{a}^{b} d x^{\prime}\left(V_{0}+\alpha\left(x^{\prime}-b\right)-E_{0}^{b}\right)^{1 / 2}} \\
& \times\left(e^{\left(V_{0}+\alpha(a-b)-E_{0}^{b}\right)^{1 / 2}(x-a)}+r e^{-\left(V_{0}+\alpha(a-b)-E_{0}^{b}\right)^{1 / 2}(x-a)}\right),
\end{aligned}
$$

with a new reflection amplitude $r$. For $x \lesssim a$ we try the left-going plane wave

$$
e(s, x)=\frac{C_{0}^{w} \cos \left(\sqrt{E_{0}^{b}}(c-b) / 2\right)\left(V_{0}-E_{0}^{b}\right)^{1 / 4}}{\left(V_{0}+\alpha(a-b)-E_{0}^{b}\right)^{1 / 4}} e^{-\int_{a}^{b} d x^{\prime}\left(V_{0}+\alpha\left(x^{\prime}-b\right)-E_{0}^{b}\right)^{1 / 2}} t e^{-i\left(E_{0}^{b}+\alpha(b-a)\right)^{1 / 2}(x-a)}
$$

with a new transmission amplitude $t$. The $C^{1}$ condition at $x=a$ gives, up to terms $\mathcal{O}(|\alpha|)$,

$$
\begin{aligned}
1+r & =t \\
\left(V_{0}+\alpha(a-b)-E_{0}^{b}\right)^{1 / 2}-\left(V_{0}+\alpha(a-b)-E_{0}^{b}\right)^{1 / 2} r & =-i\left(E_{0}^{b}-\alpha(a-b)\right)^{1 / 2} t
\end{aligned}
$$

which determines $r$ and $t$ so that for $x \lesssim a$ we have

$$
\begin{aligned}
& e(s, x)=\frac{C_{0}^{w} \cos \left(\sqrt{E_{0}^{b}}(c-b) / 2\right)\left(V_{0}-E_{0}^{b}\right)^{1 / 4}}{\left(V_{0}-\alpha(b-a)-E_{0}^{b}\right)^{1 / 4}} 2\left[1-i \frac{\left(E_{0}^{b}+\alpha(b-a)\right)^{1 / 2}}{\left(V_{0}-\alpha(b-a)-E_{0}^{b}\right)^{1 / 2}}\right]^{-1} \\
& \times \exp \left\{\frac{2}{3 \alpha}\left[\left(V_{0}-\alpha(b-a)-E_{0}^{b}\right)^{3 / 2}-\left(V_{0}-E_{0}^{b}\right)^{3 / 2}\right]-i\left(E_{0}^{b}+\alpha(b-a)\right)^{1 / 2}(x-a)\right\} .
\end{aligned}
$$

Note that for $x \lesssim a, e(s, x)$ is a true left-going plane wave only for $\Delta V$ not too large when $E_{0}^{b}+\alpha(b-a)>0$. If $E_{0}^{b}+\alpha(b-a)<0$, Eq. (5.22) becomes an exponentially decaying function whose corresponding probability current density vanishes. Since $E_{0}^{b}+\alpha(b-a)=E_{R}(s)$, this case corresponds to $E_{R}(s)<0$. In Eq. (5.22), we also assumed that $V_{0}-\alpha(b-a)-E_{0}^{b}>0$, i.e., $E_{R}(s)<V_{0}$.

The resonance width can be now computed by means of the Green formula

$$
\Gamma(s) \int_{a^{\prime}}^{d^{\prime}} d x|e(s, x)|^{2}=\left.2 \operatorname{Im}\left(\overline{e(s, x)} \partial_{x} e(s, x)\right)\right|_{a^{\prime}} ^{d^{\prime}}
$$

where $a^{\prime}<a$ and $d^{\prime}>d$. The integral in the l.h.s. of (5.23) is $1+\mathcal{O}(|\alpha|+|\beta|)$ and using (5.18) and (5.22) we get, up to such a factor,

$$
\begin{aligned}
& \Gamma(s)=8\left(C_{0}^{w}\right)^{2} E_{0}^{b}\left(V_{0}-E_{0}^{b}\right)^{1 / 2} V_{0}^{-2} \\
& \times\left[\left(V_{0}+\beta(d-c)-E_{0}^{b}\right)^{1 / 2}\left(E_{0}^{b}-\beta(d-c)\right)^{1 / 2} e^{\left[\left(V_{0}-E_{0}^{b}\right)^{3 / 2}-\left(V_{0}+\beta(d-c)-E_{0}^{b}\right)^{3 / 2}\right] 4 / 3 \beta}\right. \\
& \left.+\left(V_{0}-\alpha(b-a)-E_{0}^{b}\right)^{1 / 2}\left(E_{0}^{b}+\alpha(b-a)\right)_{+}^{1 / 2} e^{\left[\left(V_{0}-\alpha(b-a)-E_{0}^{b}\right)^{3 / 2}-\left(V_{0}-E_{0}^{b}\right)^{3 / 2}\right] 4 / 3 \alpha}\right],
\end{aligned}
$$

where we used $u_{+}=\theta(u) u$.

\section{ONE MODE APPROXIMATION}

Equation (2.13) can be simplified by developing $\tilde{\nu}$ into the instantaneous eigenstates of the potential $V+W$ and keeping only the contributions from the discrete resonant states, i.e., neglecting the contributions from the continuous spectrum [17]. For the moment, we will suppose there is only one resonant state and put $\tilde{\nu}(x, t, E)=$ $\exp (-i E t) z(t, E) e(s, x)$ where $e(s, x)$ is the (ground) resonant state of the potential $V+W$ 


$$
\left[-\lambda(s)-\partial_{x}^{2}+V(x)+W(s, x)\right] e(s, x)=0
$$

with complex eigenvalue $\lambda(s)=E_{R}(s)-i \Gamma(s) / 2$. The eigenfunction $e(s, x)$ is of class $L^{2}$ on the contour $\gamma \equiv$ $\left.\left.\left(e^{i \theta}\right]-\infty, 0\right]+a\right) \bigcup[a, d] \bigcup\left(d+e^{i \theta}[0,+\infty[)\right.$ for $\theta$ conveniently chosen [23] and satisfies

$$
\int_{\gamma} d x e(s, x)^{2}=1, \quad \int_{\gamma} d x e(s, x) \partial_{s} e(s, x)=0 .
$$

Multiplying (2.13) with $e(s, x)$ and integrating over $\gamma$, we get

$$
\partial_{t} z(t, E)=i[E-\lambda(s)] z(t, E)+\mathcal{B}(t, s, E)
$$

with the driving term given by

$$
\mathcal{B}(t, s, E)=i V_{0} \int_{b}^{c} d x \mu(x, t, E) e(s, x)
$$

and the sheet density (2.7) reduced, with small error, to

$$
s(t)=\int d E g(E)|z(t, E)|^{2} \equiv\|z(t)\|^{2} .
$$

\section{FIXED POINTS AND LINEARIZATIONS: GENERAL RESULTS}

We consider the vector field in the l.h.s. of (6.3),

$$
\mathcal{V}(z, E)=\mathcal{A}\left(\|z\|^{2}, E\right) z(E)+\mathcal{B}\left(\|z\|^{2}, E\right),
$$

where

$$
\mathcal{A}(s, E)=-\Gamma(s) / 2+i\left(E-\left(E_{R}(0)+\eta s\right)\right),
$$

is a non-vanishing function. For simplicity, we assume that $\mathcal{B}$ is independent of $t$. When $\mathcal{B}$ varies slowly with $t$, the discussion below should be applied to each such fixed value of $t$.

We first look for fixed points of $\mathcal{V}$, i.e., functions $z=z(E)$ in $L^{2}(g(E) d E)$ with $\mathcal{V}(z(E), E)=0$. Clearly $z=z(E)$ is a fixed point iff

$$
z(E)=-\frac{\mathcal{B}\left(\|z\|^{2}, E\right)}{\mathcal{A}\left(\|z\|^{2}, E\right)}
$$

so the $L^{2}$-norm $s=\|z\|^{2}$ has to satisfy

$$
s=\int d E g(E) \frac{|\mathcal{B}(s, E)|^{2}}{|\mathcal{A}(s, E)|^{2}} .
$$

Conversely, if $s \geq 0$ is a solution of (7.4), then

$$
z(E)=-\frac{\mathcal{B}(s, E)}{\mathcal{A}(s, E)}
$$

gives the unique fixed point of $\mathcal{V}$ with $\|z\|^{2}=s$.

Assuming that we have found a fixed point $z=z(E)$, we look for the linearization of the vector field $\mathcal{V}$ at that point. By giving an infinitesimal increment $\delta z(E)$ to $z(E)$, the corresponding increment $\delta \mathcal{V}$ to $\mathcal{V}$ is

$$
\delta \mathcal{V}(z, E)=\mathcal{A}(s, E) \delta z(E)+(\langle\delta z \mid z\rangle+\langle\overline{\delta z} \mid \bar{z}\rangle)\left(\partial_{s} \mathcal{A}(s, E) z(E)+\partial_{s} \mathcal{B}(s, E)\right)
$$

where $s=\|z\|^{2}$ is the corresponding solution of (7.4) and $\langle u \mid v\rangle=\int d E g(E) u(E) \overline{v(E)}$. Hence,

$$
\overline{\delta \mathcal{V}}(z, E)=\overline{\mathcal{A}}(s, E) \overline{\delta z}(E)+(\langle\delta z \mid z\rangle+\langle\overline{\delta z} \mid \bar{z}\rangle)\left(\overline{\partial_{s} \mathcal{A}}(s, E) \bar{z}(E)+\overline{\partial_{s} \mathcal{B}}(s, E)\right)
$$


so with $u(E)=\delta z(E)$ and $v(E)=\overline{\delta z}(E)$, we get the complexification of the linearization,

$$
\mathcal{L}\left(\begin{array}{l}
u \\
v
\end{array}\right)=\left(\begin{array}{cc}
\mathcal{A} & 0 \\
0 & \overline{\mathcal{A}}
\end{array}\right)\left(\begin{array}{l}
u \\
v
\end{array}\right)+\left(\begin{array}{c}
(\langle u \mid z\rangle+\langle v \mid \bar{z}\rangle)\left(\partial_{s} \mathcal{A} z+\partial_{s} \mathcal{B}\right) \\
(\langle u \mid z\rangle+\langle v \mid \bar{z}\rangle)\left(\overline{\partial_{s} \mathcal{A}} \bar{z}+\overline{\partial_{s} \mathcal{B}}\right)
\end{array}\right) .
$$

The matrix in the first term of the r.h.s. has continuous spectrum contained in $-\Gamma(s) / 2+i \boldsymbol{R}$ and the second term appears as a rank one perturbation. If $\lambda \in C$ is an eigenvalue of $\mathcal{L}$ with real part different from $-\Gamma(s) / 2$, we get

$$
\begin{aligned}
& (\mathcal{A}(s, E)-\lambda) u+(\langle u \mid z\rangle+\langle v \mid \bar{z}\rangle)\left(\partial_{s} \mathcal{A}(s, E) z+\partial_{s} \mathcal{B}(s, E)\right)=0 \\
& (\overline{\mathcal{A}}(s, E)-\lambda) v+(\langle u \mid z\rangle+\langle v \mid \bar{z}\rangle)\left(\overline{\partial_{s} \mathcal{A}}(s, E) \bar{z}+\overline{\partial_{s} \mathcal{B}}(s, E)\right)=0 .
\end{aligned}
$$

We must then have

$$
\begin{aligned}
u(E) & =\kappa \frac{\partial_{s} \mathcal{A}(s, E) z+\partial_{s} \mathcal{B}(s, E)}{\mathcal{A}(s, E)-\lambda} \\
v(E) & =\kappa \frac{\overline{\partial_{s} \mathcal{A}}(s, E) \bar{z}+\overline{\partial_{s} \mathcal{B}}(s, E)}{\overline{\mathcal{A}}(s, E)-\lambda},
\end{aligned}
$$

where $\kappa=\langle u \mid z\rangle+\langle v \mid \bar{z}\rangle$. In order to have a non-trivial solution $\kappa \neq 0$, it is necessary and sufficient that

$$
1+\int d E g(E) \frac{\partial_{s}((\mathcal{A}-\lambda)(\overline{\mathcal{A}}-\lambda))|\mathcal{B}|^{2}-\mathcal{A}(\overline{\mathcal{A}}-\lambda) \overline{\mathcal{B}} \partial_{s} \mathcal{B}-\overline{\mathcal{A}}(\mathcal{A}-\lambda) \mathcal{B} \partial_{s} \overline{\mathcal{B}}}{\left((\operatorname{Re} \mathcal{A}-\lambda)^{2}+(\operatorname{Im} \mathcal{A})^{2}\right)|\mathcal{A}|^{2}}=0 .
$$

Here, the l.h.s. is real for real $\lambda$, and tends to 1 , when $\lambda \rightarrow+\infty$.

On the other hand, the $s$-derivative of the l.h.s. minus the r.h.s. of $(7.4)$ is

$$
1+\int d E g(E) \frac{|\mathcal{B}(s, E)|^{2} \partial_{s}|\mathcal{A}(s, E)|^{2}-|\mathcal{A}(s, E)|^{2} \partial_{s}|\mathcal{B}(s, E)|^{2}}{|\mathcal{A}(s, E)|^{4}}
$$

which coincides with the l.h.s. of (7.11) for $\lambda=0$. So if the expression (7.12) is $<0$, we see that (7.11) must have a solution $\lambda>0$. Let us say that the fixed point is stable if the spectrum of the linearization $\mathcal{L}$ is contained in the open left half-plane and unstable otherwise. The discussion above then gives:

Proposition VII.1 Let $z$ be a fixed point of $\mathcal{V}$ so that (7.4) and 7.5) hold. If the s-derivative of the l.h.s. minus the r.h.s. of (7.4) is $<0$, then $z$ is an unstable fixed point. More precisely, the linearization $\mathcal{L}$ then has an eigenvalue which is $>0$.

\section{FIXED POINTS AND LINEARIZATIONS: THE SMALL-Г LIMIT}

In this section we assume that the driving term $\mathcal{B}(s, E)$ is a sufficiently smooth function of $E$, at least near the point $E_{R}(0)+\eta s$, where $s$ solves (7.4). When the barriers are very wide, $\Gamma(s)$ will be very small and

$$
\frac{1}{|\mathcal{A}(s, E)|^{2}}=\frac{1}{(\Gamma(s) / 2)^{2}+\left(E_{R}(0)+\eta s-E\right)^{2}}
$$

is a function of $E$ which is sharply peaked at $E_{R}(s)=E_{R}(0)+\eta s$. In (7.4) it is therefore justified to replace $g(E)|\mathcal{B}(s, E)|^{2}$ by the constant value $g\left(E_{R}(0)+\eta s\right)\left|\mathcal{B}\left(s, E_{R}(0)+\eta s\right)\right|^{2}$. Then (7.4) is well approximated by

$$
s=2 \pi \frac{g\left(E_{R}(s)\right)\left|\mathcal{B}\left(s, E_{R}(s)\right)\right|^{2}}{\Gamma(s)} .
$$

We shall next apply a similar argument to the equation (7.11) for the eigenvalues of the linearization $\mathcal{L}$ and, for more transparency, we start with a simplified case, in which 
In this case, (7.11) reduces to

$$
1-2 \eta \int d E \frac{\left(E-E_{R}(0)-\eta s\right) g(E)|\mathcal{B}(E)|^{2}}{\left[(\Gamma / 2+\lambda)^{2}+\left(E-E_{R}(0)-\eta s\right)^{2}\right]\left[(\Gamma / 2)^{2}+\left(E-E_{R}(0)-\eta s\right)^{2}\right]}=0 .
$$

We shall use,

$$
\begin{gathered}
\int_{-\infty}^{+\infty} d t \frac{t^{2}}{\left(q^{2}+t^{2}\right)\left(p^{2}+t^{2}\right)}= \begin{cases}\frac{\pi}{p+q}, & \operatorname{Re} p>0, \operatorname{Re} q>0 \\
\frac{\pi}{p-q}, & \operatorname{Re} p>0, \operatorname{Re} q<0\end{cases} \\
\int_{-\infty}^{+\infty} d t \frac{1}{\left(q^{2}+t^{2}\right)\left(p^{2}+t^{2}\right)}= \begin{cases}\frac{\pi}{q p(p+q)}, & \operatorname{Re} p>0, \operatorname{Re} q>0 \\
\frac{-\pi}{q p(p-q)}, & \operatorname{Re} p>0, \operatorname{Re} q<0 .\end{cases}
\end{gathered}
$$

If we replace $g(E)|\mathcal{B}(E)|^{2}$ in the integral in (8.3) with its value at $E=E_{R}(0)+\eta s$, that integral vanishes since the integrand becomes an odd function of $E-E_{R}(0)-\eta s$. Instead, we get an approximation of the integral in (8.3) by replacing $g(E)|\mathcal{B}(E)|^{2}$ with the linear term in its Taylor expansion at $E=E_{R}(0)+\eta s$. Using (8.4), we then get from (8.3)

$$
\begin{aligned}
& 1-2 \eta \frac{\left(g|\mathcal{B}|^{2}\right)^{\prime}\left(E_{R}(0)+\eta s\right) \pi}{\Gamma+\lambda}=0, \text { when } \Gamma / 2+\operatorname{Re} \lambda>0, \\
& 1+2 \eta \frac{\left(g|\mathcal{B}|^{2}\right)^{\prime}\left(E_{R}(0)+\eta s\right) \pi}{\lambda}=0, \text { when } \Gamma / 2+\operatorname{Re} \lambda<0,
\end{aligned}
$$

where $\left(g|\mathcal{B}|^{2}\right)^{\prime}=\partial_{E}\left(g|\mathcal{B}|^{2}\right)$. The solution of $(8.6)$ is

$$
\lambda=2 \pi \eta\left(g|\mathcal{B}|^{2}\right)^{\prime}\left(E_{R}(0)+\eta s\right)-\Gamma,
$$

and this is an eigenvalue of the linearization $\mathcal{L}$ as long as

$$
2 \pi \eta\left(g|\mathcal{B}|^{2}\right)^{\prime}\left(E_{R}(0)+\eta s\right)>\frac{\Gamma}{2} .
$$

The solution of (8.7) is

$$
\lambda=-2 \pi \eta\left(g|\mathcal{B}|^{2}\right)^{\prime}\left(E_{R}(0)+\eta s\right),
$$

and describes an eigenvalue of $\mathcal{L}$ precisely when (8.9) is fulfilled. We then have the following conclusion under the simplifying assumption (8.2) and in the small- $\Gamma$ limit.

When $2 \pi \eta\left(g|\mathcal{B}|^{2}\right)^{\prime}\left(E_{R}(0)+\eta s\right) \leq \Gamma / 2$ : no eigenvalues of $\mathcal{L}$ and hence an attractive fixed point.

When $\Gamma / 2<2 \pi \eta\left(g|\mathcal{B}|^{2}\right)^{\prime}\left(E_{R}(0)+\eta s\right)<\Gamma$ : two eigenvalues of $\mathcal{L}$ and still an attractive fixed point.

When $2 \pi \eta\left(g|\mathcal{B}|^{2}\right)^{\prime}\left(E_{R}(0)+\eta s\right) \geq \Gamma$ : two eigenvalues and a non-attractive fixed point.

The main conclusion under the same assumptions is then:

Proposition VIII.1 We have an attractive fixed point precisely when the s-derivative of the difference of the l.h.s. and the r.h.s. in (8.1) is $>0$.

Now we drop the simplifying assumption (8.2) and see that the preceding proposition still holds in the small- $\Gamma$ limit. Let $z$ be a fixed point, so that $s=\|z\|^{2}$ (approximately) solves (8.1). In view of (7.2), Eq. (7.11) can be written as

$$
1+\int d E g(E) \frac{\partial_{s}((\mathcal{A}-\lambda)(\overline{\mathcal{A}}-\lambda))|\mathcal{B}|^{2}-\mathcal{A}(\overline{\mathcal{A}}-\lambda) \partial_{s} \mathcal{B} \overline{\mathcal{B}}-\overline{\mathcal{A}}(\mathcal{A}-\lambda) \mathcal{B} \partial_{s} \overline{\mathcal{B}}}{\left[(\Gamma(s) / 2+\lambda)^{2}+\left(E-E_{R}(0)-\eta s\right)^{2}\right]\left[(\Gamma(s) / 2)^{2}+\left(E-E_{R}(0)-\eta s\right)^{2}\right]}=0
$$

Here, the numerator of the integrand can be simplified to

$$
\begin{aligned}
& {\left[\partial_{s} \Gamma(\Gamma / 2+\lambda)|\mathcal{B}|^{2}-(\Gamma / 2)(\Gamma / 2+\lambda) \partial_{s}|\mathcal{B}|^{2}\right]} \\
& +\left[\left(E-E_{R}(0)-\eta s\right)\left(-2 \eta|\mathcal{B}|^{2}+i \lambda\left(\partial_{s} \mathcal{B} \overline{\mathcal{B}}-\mathcal{B} \partial_{s} \overline{\mathcal{B}}\right)\right]-\left[\left(E-E_{R}(0)-\eta s\right)^{2} \partial_{s}|\mathcal{B}|^{2}\right]\right.
\end{aligned}
$$


Accordingly, we split the integral into three pieces and apply the small- $\Gamma$ approximation to each one. If we assume, for simplicity, that $\Gamma / 2+\operatorname{Re} \lambda>0$ (which is necessarily the case if the eigenvalue $\lambda$ is to ruin attractiveness) we get

$$
\begin{aligned}
1+ & \frac{\left[\partial_{s} \Gamma(\Gamma / 2+\lambda)|\mathcal{B}|^{2}-(\Gamma / 2)(\Gamma / 2+\lambda) \partial_{s}|\mathcal{B}|^{2}\right] \pi g}{(\Gamma / 2)(\Gamma / 2+\lambda)(\Gamma+\lambda)} \\
& +\frac{\left[\partial_{E}\left(g\left(-2 \eta|\mathcal{B}|^{2}+i \lambda\left(\partial_{s} \mathcal{B} \overline{\mathcal{B}}-\mathcal{B} \partial_{s} \overline{\mathcal{B}}\right)\right)\right)-g \partial_{s}|\mathcal{B}|^{2}\right] \pi}{\Gamma+\lambda}=0
\end{aligned}
$$

at $E=E_{R}(0)+\eta s$. This can be rewritten as

$$
1+\frac{2 \pi\left(\partial_{s} \Gamma / \Gamma\right) g|\mathcal{B}|^{2}}{\Gamma+\lambda}-\frac{2 \pi \partial_{s}\left(g|\mathcal{B}|^{2}\right)}{\Gamma+\lambda}-\frac{2 \pi \eta \partial_{E}\left(g|\mathcal{B}|^{2}\right)}{\Gamma+\lambda}+\frac{i \lambda \pi \partial_{E}\left(g\left(\partial_{s} \mathcal{B} \overline{\mathcal{B}}-\mathcal{B} \partial_{s} \overline{\mathcal{B}}\right)\right)}{\Gamma+\lambda}=0,
$$

again at $E=E_{R}(0)+\eta s$. Noticing that

$$
\frac{d}{d s}\left(\left(g|\mathcal{B}|^{2}\right)\left(s, E_{R}(0)+\eta s\right)\right)=\left(\eta \partial_{E}+\partial_{s}\right)\left(g|\mathcal{B}|^{2}\right)\left(s, E_{R}(0)+\eta s\right),
$$

and multiplying with $\Gamma+\lambda$, we get the following approximation of (7.11)

$$
\begin{aligned}
& \lambda\left[1+i \pi \partial_{E}\left(g\left(\partial_{s} \mathcal{B} \overline{\mathcal{B}}-\mathcal{B} \partial_{s} \overline{\mathcal{B}}\right)\right)\right] \\
& \quad=-\Gamma(s)-2 \pi \frac{\left(g|\mathcal{B}|^{2}\right)\left(s, E_{R}(0)+\eta s\right)}{\Gamma(s)} \partial_{s} \Gamma(s)+2 \pi \frac{d}{d s}\left(\left(g|\mathcal{B}|^{2}\right)\left(s, E_{R}(0)+\eta s\right)\right) .
\end{aligned}
$$

We assume that $1+i \pi \partial_{E}\left(g\left(\partial_{s} \mathcal{B} \overline{\mathcal{B}}-\mathcal{B} \partial_{s} \overline{\mathcal{B}}\right)\right)>0$, so that the solution $\lambda$ of $(8.15)$ is real and has the same sign as the r.h.s. of (8.15).

On the other hand, the $s$-derivative of the l.h.s. minus the r.h.s. of 8.1) is

$$
\begin{aligned}
& 1-\frac{2 \pi}{\Gamma(s)} \frac{d}{d s}\left(\left(g|\mathcal{B}|^{2}\right)\left(s, E_{R}(0)+\eta s\right)\right)+2 \pi\left(g|\mathcal{B}|^{2}\right)\left(s, E_{R}(0)+\eta s\right) \frac{\partial_{s} \Gamma(s)}{\Gamma(s)^{2}} \\
& =-\frac{1}{\Gamma(s)}\left(-\Gamma(s)+2 \pi \frac{d}{d s}\left(\left(g|\mathcal{B}|^{2}\right)\left(s, E_{R}(0)+\eta s\right)\right)-2 \pi \frac{\left(g|\mathcal{B}|^{2}\right)\left(s, E_{R}(0)+\eta s\right)}{\Gamma(s)} \partial_{s} \Gamma(s)\right),
\end{aligned}
$$

which is of the opposite sign to the r.h.s. in (8.15). We then have:

Proposition VIII.2 Under the weaker assumptions above and in the small- $\Gamma$ limit, we still have an attractive fixed point precisely when the s-derivative of the l.h.s. minus the r.h.s. of (8.1) is $>0$.

\section{A SIMPLIFIED DIFFERENTIAL EQUATION FOR THE SHEET DENSITY}

Consider the differential equation (6.3)

$$
\partial_{t} z(t, E)=\left[-\Gamma(s(t)) / 2+i\left(E-\left(E_{R}(0)+\eta s(t)\right)\right)\right] z(t, E)+\mathcal{B}(s(t), E),
$$

where $s(t)=\|z(t, \cdot)\|^{2}$, and where we could also let $\mathcal{B}$ be a slowly varying function of $t$ through $s(t)$. Assuming $s(t)$ to be a known function, the solution of (9.1) with a prescribed initial value at time $t_{0}$ is

$$
\begin{array}{r}
z(t, E)=\int_{t_{0}}^{t} d t^{\prime} e^{i\left(E-E_{R}(0)\right)\left(t-t^{\prime}\right)-\int_{t^{\prime}}^{t} d t^{\prime \prime} \Gamma\left(s\left(t^{\prime \prime}\right)\right) / 2-i \eta \int_{t^{\prime}}^{t} d t^{\prime \prime} s\left(t^{\prime \prime}\right)} \mathcal{B}\left(s\left(t^{\prime}\right), E\right) \\
+e^{i\left(E-E_{R}(0)\right)\left(t-t_{0}\right)-\int_{t_{0}}^{t} d t^{\prime} \Gamma\left(s\left(t^{\prime}\right)\right) / 2-i \eta \int_{t_{0}}^{t} d t^{\prime} s\left(t^{\prime}\right)} z\left(t_{0}, E\right) .
\end{array}
$$

Assuming that the solution has existed as a bounded solution for a very long time, say from the time $-\infty$, we can let $t_{0}$ tend to $-\infty$ in the formula above and get

$$
z(t, E)=\int_{-\infty}^{t} d t^{\prime} e^{i\left(E-E_{R}(0)\right)\left(t-t^{\prime}\right)-\int_{t^{\prime}}^{t} d t^{\prime \prime} \Gamma\left(s\left(t^{\prime \prime}\right)\right) / 2-i \eta \int_{t^{\prime}}^{t} d t^{\prime \prime} s\left(t^{\prime \prime}\right)} \mathcal{B}\left(s\left(t^{\prime}\right), E\right) .
$$


Taking the scalar product of 9.1 ) and $z$ gives the following equation for the derivative of the sheet density

$$
\frac{d}{d t} s(t)=2 \operatorname{Re}\left\langle z \mid \partial_{t} z\right\rangle=-\Gamma(s(t)) s(t)+2 \operatorname{Re}\langle z \mid \mathcal{B}\rangle,
$$

where

$$
\begin{aligned}
& 2 \operatorname{Re}\langle z \mid \mathcal{B}\rangle= \\
& 2 \operatorname{Re} \int d E g(E) \int_{-\infty}^{t} d t^{\prime} e^{i\left(E-E_{R}(0)\right)\left(t-t^{\prime}\right)-\int_{t^{\prime}}^{t} d t^{\prime \prime} \Gamma\left(s\left(t^{\prime \prime}\right)\right) / 2-i \eta \int_{t^{\prime}}^{t} d t^{\prime \prime} s\left(t^{\prime \prime}\right)} \mathcal{B}\left(s\left(t^{\prime}\right), E\right) \overline{\mathcal{B}(s(t), E)} .
\end{aligned}
$$

We now assume that $s(t)$ varies slowly with $t$ and replace $\mathcal{B}\left(s\left(t^{\prime}\right), E\right)$ in the above integral by $\mathcal{B}(s(t), E)$. Making the $E$-integration first, we get

$$
2 \operatorname{Re}\langle z \mid \mathcal{B}\rangle=2 \operatorname{Re} \int_{-\infty}^{t} d t^{\prime} e^{-i E_{R}(0)\left(t-t^{\prime}\right)-\int_{t^{\prime}}^{t} d t^{\prime \prime} \Gamma\left(s\left(t^{\prime \prime}\right)\right) / 2-i \eta \int_{t^{\prime}}^{t} d t^{\prime \prime} s\left(t^{\prime \prime}\right)} \mathcal{F}\left(g|\mathcal{B}|^{2}\right)\left(s(t), t^{\prime}-t\right),
$$

where $\mathcal{F}$ denotes the Fourier transform with respect to $E$. Assuming $g(E)|\mathcal{B}(s(t), E)|^{2}$ sufficiently smooth as a function of $E$, we see that $\mathcal{F}\left(g|\mathcal{B}|^{2}\right)\left(s(t), t^{\prime}-t\right)$ is sufficiently rapidly decreasing as a function of $t^{\prime}-t$ for the following approximations to be made: i) since $\Gamma(s)$ is small, we may assume that $\exp \left\{-\int_{t^{\prime}}^{t} d t^{\prime \prime} \Gamma\left(t^{\prime \prime}\right) / 2\right\} \simeq 1$ ii) since $s\left(t^{\prime \prime}\right)$ varies slowly, we may replace $\int_{t^{\prime}}^{t} d t^{\prime \prime} s\left(t^{\prime \prime}\right)$ by $s(t)\left(t-t^{\prime}\right)$. We then get

$$
\begin{aligned}
2 \operatorname{Re}\langle z \mid \mathcal{B}\rangle & \simeq 2 \operatorname{Re} \int_{-\infty}^{t} d t^{\prime} e^{-i\left(E_{R}(0)+\eta s(t)\right)\left(t-t^{\prime}\right)} \mathcal{F}\left(g|\mathcal{B}|^{2}\right)\left(s(t), t^{\prime}-t\right) \\
& =2 \operatorname{Re} \int_{-\infty}^{0} d t^{\prime} e^{i\left(E_{R}(0)+\eta s(t)\right) t^{\prime}} \mathcal{F}\left(g|\mathcal{B}|^{2}\right)\left(s(t), t^{\prime}\right)
\end{aligned}
$$

Using the property $\mathcal{F}(u)(-t)=\overline{\mathcal{F}(u)(t)}$, valid for any real valued function $u(E)$, we obtain

$$
2 \operatorname{Re}\langle s \mid \mathcal{B}\rangle=\int_{-\infty}^{+\infty} d t^{\prime} e^{i\left(E_{R}(0)+\eta s(t)\right) t^{\prime}} \mathcal{F}\left(g|\mathcal{B}|^{2}\right)\left(s(t), t^{\prime}\right)=2 \pi\left(g|\mathcal{B}|^{2}\right)\left(s(t), E_{R}(0)+\eta s(t)\right) .
$$

Inserting this in (9.3), we get the approximate differential equation for the sheet density $s(t)=\|z(t, \cdot)\|^{2}$

$$
\frac{d}{d t} s(t)=-\Gamma(s(t))\left[s(t)-2 \pi \frac{\left(g|\mathcal{B}|^{2}\right)\left(s(t), E_{R}(0)+\eta s(t)\right)}{\Gamma(s(t))}\right] .
$$

This equation is valid for slowly varying solutions which have evolved for a time much longer than $\Gamma^{-1}$.

\section{QUALITATIVE DISCUSSION AND NUMERICAL RESULTS}

We start by examining the simplified fixed point equation (8.1). For $0 \leq E \lesssim E_{F}$ with $E_{F} \ll V_{0}$, we have $V_{0}-E \sim V_{0}$ (of the same order of magnitude). By evaluating the integral in (6.4) with $e(s, x)$ approximated by (5.2) and the driving term given by (4.7), we have

$$
|\mathcal{B}(s, E)|^{2} \sim\left(C_{0}^{w}\right)^{2} V_{0}^{-1} E_{0}^{w} E^{1 / 2} e^{\left[\left(V_{0}-E\right)^{3 / 2}-\left(V_{0}+\alpha(b-a)-E\right)^{3 / 2}\right] 4 / 3 \alpha} .
$$

Assuming for simplicity zero temperature, so that $g(E)=\theta(E)\left(E_{F}-E\right)_{+} / 2 \pi$, we get

$$
\begin{aligned}
& g\left(E_{R}(s)\right)\left|\mathcal{B}\left(s, E_{R}(s)\right)\right|^{2} \\
& \quad \sim\left(C_{0}^{w}\right)^{2} V_{0}^{-1} E_{0}^{w} E_{R}(s)_{+}^{1 / 2}\left(E_{F}-E_{R}(s)\right)_{+} e^{\left[\left(V_{0}-E_{R}(s)\right)^{3 / 2}-\left(V_{0}-E_{R}(s)+\alpha(b-a)\right)^{3 / 2}\right] 4 / 3 \alpha} .
\end{aligned}
$$

Recalling that $E_{R}(s)=E_{R}(0)+\eta s=E_{0}^{b}+\alpha(b-a)=E_{0}^{b}-\beta(d-c)-\Delta V$, where $\alpha$ and $\beta$ are given by (4.3) and (5.6), respectively, from 5.24) we get 


$$
\begin{aligned}
& \Gamma(s) \sim\left(C_{0}^{w}\right)^{2} E_{0}^{b} V_{0}^{-3 / 2}\left[\left(V_{0}-E_{R}(s)\right)^{1 / 2} E_{R}(s)_{+}^{1 / 2} e^{\left[\left(V_{0}-E_{R}(s)\right)^{3 / 2}-\left(V_{0}-E_{R}(s)+\alpha(b-a)\right)^{3 / 2}\right] 4 / 3 \alpha}\right. \\
& \left.+\left(V_{0}-\Delta V-E_{R}(s)\right)^{1 / 2}\left(E_{R}(s)+\Delta V\right)^{1 / 2} e^{\left[\left(V_{0}-\Delta V-E_{R}(s)-\beta(d-c)\right)^{3 / 2}-\left(V_{0}-\Delta V-E_{R}(s)\right)^{3 / 2}\right] 4 / 3 \beta}\right] .
\end{aligned}
$$

We will consider the following two cases:

1) The barrier $[c, d]$ is more opaque than $[a, b]$ in the sense that the exponential factor in the second term of the above expression for $\Gamma(s)$ is much smaller than the exponential factor in the first term.

2) The barrier $[a, b]$ is more opaque than $[c, d]$.

In the intermediate case when the two barriers have opacity of the same order, the discussion of case 1) will roughly apply. Notice that opacity depends not only on the relative sizes of $b-a$ and $d-c$, but also on $s$ and $\Delta V$. Therefore, we may have transitions between the two cases when these parameters vary. Interesting phenomena appear when the case 1$)$ is possible and we start with that case, recalling that $E_{R}(s)=E_{R}(0)+\eta s=E_{0}^{w}-\Delta V(b-a) / \ell+\eta s$. In this case (and neglecting, to start with, the possibility of a transition to the case 2)) the first term in the expression for $\Gamma(s)$ dominates, except when $E_{R}(s)$ is negative or very small and positive. The function

$$
f(s)=2 \pi \frac{g\left(E_{R}(s)\right)\left|\mathcal{B}\left(s, E_{R}(s)\right)\right|^{2}}{\Gamma(s)}
$$

vanishes for $E_{R}(s) \leq 0$ and rises very sharply (with a square root singularity at $E_{R}(s)=0$ ) from 0 to

$$
f_{\max } \sim E_{F}
$$

when $E_{R}(s)$ is increased from 0 to a small positive value. When $E_{R}(s)$ is further increased, the function $f(s)$ decreases roughly linearly and vanishes for $E_{R}(s) \geq E_{F}$. The values $E_{R}(s)=0, E_{R}(s)=E_{F}$ correspond to

$$
s=\left(\Delta V(b-a) / \ell-E_{0}^{w}\right) / \eta, \quad s=\left(\Delta V(b-a) / \ell-E_{0}^{w}+E_{F}\right) / \eta,
$$

and describe the boundary points of the support of the function (10.1). When $\Delta V$ is increased, these two points move to the right with the same speed as shown in the example of Fig. 2. In Fig. 2 we also see the graphical solution of Eq. (8.1), $s=f(s)$, for different values of $\Delta V$. It is clear that (8.1) will first have only one solution when $\Delta V(b-a) / \ell-E_{0}^{w} \leq 0$, then three solutions for $\Delta V$ in some interval, until $\Delta V(b-a) / \ell-E_{0}^{w} \sim \eta f_{\max }$, and again only one solution for even larger values of $\Delta V$. According to the results of section VIII, we see that in the case in which (8.1) has only one solution, this solution corresponds to an attractive fixed point, and when there are three solutions, the smallest and the largest of these correspond to attractive fixed points, while the intermediate solution corresponds to an unstable fixed point.

For many experimentally relevant situations the resonance width is much smaller than the other energy scales (essentially $E_{F}$ ). In this case we may expect the simplified fixed point equation (3.1) to be a very good approximation of the more correct equation (7.4), except near the boundary points of the support of the function (10.1). This is confirmed by Fig. 3 where we show the numerical solutions (stable and unstable) of both (7.4) and (8.1) for a system having $\Gamma(0) / E_{F} \simeq 0.01$ at $\Delta V=0.2 \mathrm{eV}$. In the case of Eq. (7.4), the corresponding energy integral has been evaluated on a uniform energy mesh having a density of points $\gg \Gamma(0)^{-1}$.

The solutions of the simplified differential equation (9.7) converge to one of the solutions of (8.1), associated to an attractive fixed point. The phenomenon of hysteresis then becomes clear. We let $\Delta V$ increase very slowly from some sufficiently small value up to some sufficiently large positive value and subsequently decrease it very slowly, back to its initial value. Consider a corresponding solution of the time dependent Schrödinger equation (2.3) so that we expect the corresponding evolution of the sheet density to be well approximated by the solution of (9.7), where $\mathcal{B}$ varies slowly with time. First, there is only one (attractive) fixed point and the time dependent solution has to stay close to that fixed point. Then we have creation of a pair of fixed points (one stable and one unstable) away from the solution, but the solution continues to stay close to the old (stable) fixed point. When $\Delta V$ reaches a sufficiently large value, the unstable fixed point runs into the old stable one and they both disappear. At this point, the time dependent solution has no other choice than to converge to the only remaining fixed point (which is stable). When decreasing $\Delta V$ back to its initial value, we have the same behaviour, in the sense that the solution stays close to the initially unique fixed point as long as it exists and converges to the new unique fixed point after the old one has collapsed with the unstable one. The bias energy $\Delta V$ at which this collapse happens depends on the value of the time dependent solution and therefore is different when $\Delta V$ is increased or decreased.

The phenomenon of hysteresis is clearly seen in Fig. 3, where the collapse points for $\Delta V$ decreased from large values and $\Delta V$ increased from small values have been marked with $A$ and $B$, respectively. We have $\Delta V_{A}<\Delta V_{B}$. We 
can estimate the order of magnitude of the hysteresis width $\Delta V_{B}-\Delta V_{A}$ by considering that $\Delta V_{A}$ is determined by the condition $E_{R}(s=0)=0$ and $\Delta V_{B}$ by the condition $E_{R}\left(s \simeq f_{\max }\right) \simeq 0$. We have

$$
\Delta V_{B}-\Delta V_{A} \sim \eta f_{\max } \ell(b-a)^{-1} \sim a_{B}^{-1} E_{F}(d-c) .
$$

In Figs. 4 and 5 we show the time dependent evolution of the sheet density $s(t)$ when we start from a fixed point solution corresponding to the point $A$ or $B$ and give an instantaneous small decrement or increment $\delta V$ to $\Delta V_{A}$ or $\Delta V_{B}$, respectively. In these figures, the thick lines are the solutions of the full Schrödinger equation (6.3) and (6.5) and the thin lines the solution of the simplified differential equation (9.7). In Fig. 4 the solutions corresponding to the small- $\Gamma$ limit and the full Schrödinger equation start, as shown in Fig. 3, from different fixed point values, $s(0)$, and converge to the same (approximatively) values. On the other hand, when the starting point is $B$ (Fig. 5) the small- $\Gamma$ approximation is close to the solution of the full equation except for the value which $s(t)$ has to converge to, again in agreement with Fig. 3.

As a third example of time evolution of the sheet density of electrons in the well, in Fig. 6 we show the behavior of $s(t)$ solution of the full Schrödinger equation (6.3) and (6.5) after an instantaneous change $\delta V$ of the bias energy corresponding to the point $C$ of Fig. 3 well inside the hysteresis region. If $|\delta V|$ is chosen sufficiently large, we observe oscillations of $s(t)$ on the picosecond time scale. Contrary to the claim of [14], these oscillations are damped since $s(t)$ has to converge to the fixed point solution corresponding the bias energy $\Delta V_{C}+\delta V$.

In the case 2), when the barrier $[a, b]$ is more opaque than the barrier $[c, d]$, the function $(10.1)$ is very small, and for solutions of (8.1) we can observe only a microscopical hysteresis effect, due to the square root singularity at $E_{R}(s)=0$, which is likely to be completely absent in the more correct equation (7.4). The absence of the hysteresis effect in this case is in agreement with the experimental results of [18] and is discussed in [16].

Let us finally consider the case of very wide barriers and see that a transition between the cases 1) and 2) has to take place in the hysteresis region. Let $c-b=$ constant, $(b-a) /(d-c)=$ constant $<1$, and $b-a \rightarrow \infty$. In this limit, $\eta \sim a_{B}^{-1}(b-a)$, and the values in (10.3) are the endpoints of a short interval of length $\sim E_{F} a_{B} /(b-a)$. Let us consider (8.1) when $\Delta V$ is increased from the initial value $\left(E_{0}^{w}-E_{F}\right) \ell /(b-a)$ for which the right end point in (10.3) is 0 . If the constant $(b-a) /(d-c)$ is sufficiently small, we are in the case 1). For $\left(E_{0}^{w}-E_{F}\right) \ell /(b-a) \leq \Delta V \leq E_{0}^{w} \ell /(b-a)$, we remain in the case 1$)$, provided that $(b-a) /(d-c)$ is sufficiently small, and (8.1) has a unique solution. At $\Delta V=E_{0}^{w} \ell /(b-a)$ we have the creation of two new fixed points. If we follow the old fixed point, we cannot remain in the case 1) until it disappears. Indeed, if we did, the disappearance would take place when $s \sim f_{\max }$ and at a bias energy $\Delta V \sim a_{B}^{-1}(d-c) f_{\max }$ obtained by the condition $E_{R}\left(s \simeq f_{\max }\right) \simeq 0$. Since $E_{R}(s)=E_{0}^{b}+\alpha(b-a)$ is between 0 and $E_{F}$, the inclination $\alpha$ of the first barrier would have to be very small and we get a finite inclination $\beta \sim-a_{B}^{-1} f_{\max }$ for the barrier $[c, d]$. Therefore, when $b-a \rightarrow \infty$ only the opacity of the first barrier would diverge and, at some point, we would be no more in the case 1). What will actually happen is that when $\Delta V$ reaches some value which is bounded independently of $b-a$, we have a transition from the case 1) to the case 2$)$ and $f_{\max }$ decreases to some value which is much smaller than the r.h.s. in (10.2). This will cause the disappearance of the fixed point for a much smaller value of $s$. When a transition from case 1) to case 2) happens, we still observe a hysteresis phenomenon, but this is now caused not only by the translation of $f(s)$ as a function of $\Delta V$ but also by a variation of its height. This effect is already apparent in Fig. 3 where we see a decreasing of the height of $f(s)$ when increasing $\Delta V$.

\section{THE CASE OF SEVERAL RESONANCES}

In this section we discuss very briefly the case with several shape resonances. Much of the discussion is similar to the case of one resonance and we shall assume that we are in a parameter range where all the WKB considerations apply.

First we review the approximation for the shape resonances. We start with the potential $V_{w}$ and consider its eigenstates $e_{j}^{w}(x), j=0,1, \ldots, N-1$ and the corresponding eigenvalues $0<E_{0}^{w}<E_{1}^{w}<\ldots<E_{N-1}^{w}<V_{0}$. Since $e_{j}^{w}(x)$ is even as a function of $x-(b+c) / 2$ for even $j$ and odd for odd $j$, we have

$$
e_{j}^{w}(x)=C_{j}^{w}\left\{\begin{array}{lr}
\sin \left((j+1) \pi / 2-\sqrt{E_{j}^{w}}(c-b) / 2\right) e^{-\left(V_{0}-E_{j}^{w}\right)^{1 / 2}(b-x)} & x<b \\
\sin \left((j+1) \pi / 2+\sqrt{E_{j}^{w}}(x-(b+c) / 2)\right) & b<x<c \\
\sin \left((j+1) \pi / 2+\sqrt{E_{j}^{w}}(c-b) / 2\right) e^{-\left(V_{0}-E_{j}^{w}\right)^{1 / 2}(x-c)} & x>c .
\end{array}\right.
$$

The $C^{1}$ condition at $x=b$, or equivalently at $x=c$, gives the quantization condition

$$
\tan \left((j+1) \pi / 2+\sqrt{E_{j}^{w}}(c-b) / 2\right)=-\left(V_{0} / E_{j}^{w}-1\right)^{-1 / 2},
$$


which can also be written as

$$
\tan \left(\sqrt{E_{j}^{w}}(c-b) / 2-j \pi / 2\right)=\left(V_{0} / E_{j}^{w}-1\right)^{1 / 2} .
$$

Representing this equation graphically, we see that $N-1$ is the largest integer $\geq 1$ with $\sqrt{V_{0}}(c-b) / 2>(N-1) \pi / 2$. The functions $e_{j}^{w}(x)$ are normalized, if we choose

$$
C_{j}^{w}=\left(\frac{E_{j}^{w}}{V_{0}\left(V_{0}-E_{j}^{w}\right)^{1 / 2}}+\frac{(c-b)}{2}+\frac{\left(V_{0}-E_{j}^{w}\right)^{1 / 2}}{V_{0}}\right)^{-1 / 2} .
$$

The eigenvalues $E_{j}^{b}$ associated to the potential $V_{b}$ in (5.5) can be studied as before, and we get

$$
E_{j}^{b}=E_{j}^{w}-8 \pi a_{B}^{-1} s \frac{\left(C_{j}^{w}\right)^{2} E_{j}^{w}}{4 V_{0}\left(V_{0}-E_{j}^{w}\right)}+\mathcal{O}\left(\alpha^{2}+\beta^{2}\right)
$$

In the following, we neglect the error $\mathcal{O}\left(\alpha^{2}+\beta^{2}\right)$. The shape resonances $\lambda_{j}(s)=E_{R, j}(s)-i \Gamma_{j}(s) / 2$ for the potential $V+W$ are then given by

$$
E_{R, j}(s)=E_{R, j}(0)+\eta_{j} s
$$

where

$$
E_{R, j}(0)=E_{j}^{w}-\Delta V(b-a) / \ell, \quad \eta_{j}=\frac{8 \pi a_{B}^{-1}(b-a)(d-c)}{b-a+d-c}-\frac{8 \pi a_{B}^{-1}\left(C_{j}^{w}\right)^{2} E_{j}^{w}}{4 V_{0}\left(V_{0}-E_{j}^{w}\right)}
$$

and

$$
\begin{aligned}
& \Gamma_{j}(s)=8\left(C_{j}^{w}\right)^{2} E_{j}^{b}\left(V_{0}-E_{j}^{b}\right)^{1 / 2} V_{0}^{-2} \\
& \times\left[\left(V_{0}+\beta(d-c)-E_{j}^{b}\right)^{1 / 2}\left(E_{j}^{b}-\beta(d-c)\right)^{1 / 2} e^{\left[\left(V_{0}-E_{j}^{b}\right)^{3 / 2}-\left(V_{0}+\beta(d-c)-E_{j}^{b}\right)^{3 / 2}\right] 4 / 3 \beta}\right. \\
& \left.+\left(V_{0}-\alpha(b-a)-E_{j}^{b}\right)^{1 / 2}\left(E_{j}^{b}+\alpha(b-a)\right)_{+}^{1 / 2} e^{\left[\left(V_{0}-\alpha(b-a)-E_{j}^{b}\right)^{3 / 2}-\left(V_{0}-E_{j}^{b}\right)^{3 / 2}\right] 4 / 3 \alpha}\right] .
\end{aligned}
$$

The corresponding resonant state $e_{j}(s, x)$, satisfying $(6.2)$, can be described as in section $\mathrm{V}$.

We still try to solve (2.11) in two steps. Equation (2.12) is treated as before, while the Eq. (2.13) is now handled by letting $\widetilde{\nu}$ be a linear combination of the $N$ resonant states $e_{0}(s, x), \ldots, e_{N-1}(s, x)$. More precisely, we write $\widetilde{\nu}(x, t, E)=\exp (-i E t) \nu(x, t, E)$ and $\widetilde{\mu}(x, t, E)=\exp (-i E t) \mu(x, t, E)$, so that (2.13) becomes

$$
\left[-i \partial_{t}-\partial_{x}^{2}+V(x)+W(s, x)-E\right] \nu(x, t, E)=V_{0} 1_{[b, c]}(x) \mu(x, t, E) .
$$

Assume,

$$
\nu(x, t, E)=\sum_{k=0}^{N-1} z_{k}(t, E) e_{k}(s, x)
$$

where $s$ is defined in (2.7) and hence will be time dependent. The functions $e_{0}(s, x), \ldots, e_{N-1}(s, x)$ approximately form an orthonormal family in $L^{2}([(a+b) / 2,(c+d) / 2])$, and if we assume that $\nu$ dominates over $\mu$ in $[(a+b) / 2,(c+d) / 2]$ then, with a small error, we have

$$
s(t)=\sum_{k=0}^{N-1}\left\|z_{k}(t, \cdot)\right\|^{2}=\|z(t, \cdot)\|^{2},
$$

where the norms are in $L^{2}(g(E) d E)$ and in $L^{2}(g(E) d E)^{N}$, respectively.

Substituting (11.8) into (11.7), multiplying by $e_{j}(s, x)$ and integrating over the contour $\gamma$, we get 


$$
\sum_{k=0}^{N-1} \int_{\gamma} d x\left[-i \partial_{t}+\lambda_{k}(s)-E\right]\left(z_{k}(t, E) e_{k}(s, x)\right) e_{j}(s, x)=V_{0} \int_{b}^{c} d x \mu(x, t, E) e_{j}(s, x) .
$$

From the relations $\int_{\gamma} d x e_{k}(s, x) e_{j}(s, x)=\delta_{k, j}$, we conclude that $\int_{\gamma} d x\left(\partial_{s} e_{k}(s, x)\right) e_{j}(s, x)$ is an anti-symmetric matrix, and since $e_{k}(s, x)$ are approximately real functions near $[b, c]$, this matrix is also very close to a real one. Equation (11.10) can be written as

$$
\begin{array}{r}
{\left[-i \partial_{t}+\lambda_{j}(s)-E\right] z_{j}(t, E)-i \partial_{t}(s(t)) \sum_{k=0}^{N-1} \int_{\gamma} d x\left(\partial_{s} e_{k}(s, x)\right) e_{j}(s, x)} \\
=V_{0} \int_{b}^{c} d x \mu(x, t, E) e_{j}(s, x) .
\end{array}
$$

Due to the facts that i) $\partial_{t} s(t)$ can be expected to be very small and ii) $e_{k}(s, x)$ is roughly independent of $s$ near $[b, c]$ so that the integral $\int_{\gamma} d x\left(\partial_{s} e_{k}(s, x)\right) e_{j}(s, x)$ can be expected to be very small, we will neglect the sum in the l.h.s. of (11.11). In this case, we have

$$
\partial_{t} z_{j}(t, E)=\left[-\Gamma_{j}(s) / 2+i\left(E-E_{R, j}(s)\right)\right] z_{j}(t, E)+\mathcal{B}_{j}(t, s, E),
$$

where $\mathcal{B}_{j}(t, s, E)=i V_{0} \int_{b}^{c} d x \mu(x, t, E) e_{j}(s, x)$.

We assume that $\mathcal{B}_{j}$ vary slowly with $t$, so it is meaningful to look at instantaneous fixed points of the vector field defined by the r.h.s. of (11.12) in $L^{2}(g(E) d E)^{N}$. Assuming, for simplicity, that $\mathcal{B}_{j}$ are independent of $t$ we see that $z(E)=\left(z_{0}(E), \ldots, z_{N-1}(E)\right)$ is a fixed point precisely when:

$$
z_{j}(E)=\frac{-\mathcal{B}_{j}(s, E)}{-\Gamma_{j}(s) / 2+i\left(E-E_{R, j}(s)\right)}, \quad j=0, \ldots, N-1,
$$

from which we get the compatibility condition for $s=\|z\|^{2}$

$$
s-\sum_{j=0}^{N-1} \int d E \frac{g(E)\left|\mathcal{B}_{j}(s, E)\right|^{2}}{\left(\Gamma_{j}(s) / 2\right)^{2}+\left(E-E_{R, j}(s)\right)^{2}}=0 .
$$

Conversely, if $s$ is a solution of (11.14), then (11.13) defines the unique fixed point with $\|z\|^{2}=s$.

In the small- $\Gamma$ limit, as in section VIII we get the simplified fixed point equation

$$
s-\sum_{j=0}^{N-1} 2 \pi \frac{\left(g\left|\mathcal{B}_{j}\right|^{2}\right)\left(s, E_{R, j}(s)\right)}{\Gamma_{j}(s)}=0 .
$$

In view of (11.4), the term of index $j$ in (11.15) is a function of $s$ with support in the interval

$$
\left(\Delta V(b-a) / \ell-E_{j}^{w}\right) / \eta_{j} \leq s \leq\left(\Delta V(b-a) / \ell-E_{j}^{w}+E_{F}\right) / \eta_{j},
$$

and when $\Delta V$ increases this interval moves to the right with speed $(b-a) /\left(\ell \eta_{j}\right)$ as shown in the example of Fig. 7.

In Fig. 8 we compare the corresponding fixed point solutions obtained by solving (11.14) with those obtained in the small- $\Gamma$ limit (11.15) as a function of the bias energy $\Delta V$. Between the points marked as $A$ and $B$ we observe five fixed points. Below we give some results about the nature of fixed points, which are more complicated than in the case of a single resonance and it is not clear that those results are applicable in the situation of Fig. 8. If we assume that they are applicable, then three fixed points are stable and two unstable. The existence of more than three fixed points, i.e., the maximum number allowed for $N=1$, is related to the possibility that the intervals (11.16) are not disjoint, as clearly understood by Fig. 7 .

It is interesting to study the evolution of the sheet density $s(t)$ away from a point like $B$ in Fig. 8 where a (presumably) stable fixed point and an unstable one collapse while two other fixed points survive. In Fig. 9 we show the behavior of $s(t)$ obtained by numerically integrating Eq. (11.12) after an instantaneous increase $\delta V$ of the initial bias $\Delta V_{B}$. If the total bias $\Delta V_{B}+\delta V<\Delta V_{C}$, where $C$ is the next point where a new couple of fixed points collapse, $s(t)$ converges to the fixed point closest to its initial value $s(0)$. When $\Delta V_{B}+\delta V>\Delta V_{C}, s(t)$ first approaches the value corresponding to the collapse point $C$ but finally has to converge to the lower unique fixed point corresponding to the chosen bias. 
Next we study the linearization of the vector field defined by the r.h.s. of (11.12) at a fixed point under the following simplifying assumption:

$\Gamma_{j}$ is independent of $s, \quad \mathcal{B}_{j}=\mathcal{B}_{j}(E)$ is independent of $t, s$,

$\eta_{j}=\eta$ is independent of $j$.

Then (11.12) becomes,

$$
\partial_{t} z_{j}(t, E)=\left[-\Gamma_{j} / 2+i\left(E-E_{R, j}(0)-\eta s\right)\right] z_{j}(t, E)+\mathcal{B}_{j}(E) .
$$

The same calculations as in section VIII show that the complexification $\mathcal{L}$ of the linearization of the vector field defined by the r.h.s. of (11.18) at a fixed point, is given by

$$
\mathcal{L}\left(\begin{array}{c}
u_{0} \\
\vdots \\
u_{N-1} \\
v_{0} \\
\vdots \\
v_{N-1}
\end{array}\right)=\left(\begin{array}{c}
{\left[-\Gamma_{0} / 2+i\left(E-E_{R, 0}(0)-\eta s\right)\right] u_{0}-i \eta(\langle u \mid z\rangle+\langle v \mid \bar{z}\rangle) z_{0}} \\
\vdots \\
{\left[-\Gamma_{N-1} / 2+i\left(E-E_{R, N-1}(0)-\eta s\right)\right] u_{N-1}-i \eta(\langle u \mid z\rangle+\langle v \mid \bar{z}\rangle) z_{N-1}} \\
{\left[-\Gamma_{0} / 2-i\left(E-E_{R, 0}(0)-\eta s\right)\right] v_{0}+i \eta(\langle u \mid z\rangle+\langle v \mid \bar{z}\rangle) \bar{z}_{0}} \\
\vdots \\
{\left[-\Gamma_{N-1} / 2-i\left(E-E_{R, N-1}(0)-\eta s\right)\right] v_{N-1}+i \eta(\langle u \mid z\rangle+\langle v \mid \bar{z}\rangle) \bar{z}_{N-1}}
\end{array}\right)
$$

Here, $\langle u \mid z\rangle=\sum_{j=0}^{N-1}\left\langle u_{j} \mid z_{j}\right\rangle_{L^{2}(g(E) d E)}$. The operator $\mathcal{L}$ is a rank one perturbation of an operator with essential spectrum contained in $\cup_{j=0}^{N-1}\left(-\Gamma_{j} / 2+i \boldsymbol{R}\right)$. We look for eigenvalues $\lambda \in \boldsymbol{C}$ with $\operatorname{Re} \lambda \neq-\Gamma_{j} / 2$ for all $j$. If $\left(u_{0}, \ldots, u_{N-1}, v_{0}, \ldots, v_{N-1}\right)$ is a corresponding eigenvector, we get as in section VIII

$$
u_{j}=\frac{\kappa z_{j}(E)}{-\Gamma_{j} / 2+i\left(E-E_{R, j}(0)-\eta s\right)-\lambda}, \quad v_{j}=\frac{-\kappa \bar{z}_{j}(E)}{-\Gamma_{j} / 2-i\left(E-E_{R, j}(0)-\eta s\right)-\lambda},
$$

where

$$
\kappa=i \eta(\langle u \mid z\rangle+\langle v \mid \bar{z}\rangle) .
$$

Using (11.13), 11.20) in (11.21), we see that $\lambda$ is an eigenvector precisely when

$$
1-2 \eta \sum_{k=0}^{N-1} \int d E \frac{\left(E-E_{R, k}(0)-\eta s\right) g(E)\left|\mathcal{B}_{k}(E)\right|^{2}}{\left[\left(\Gamma_{k} / 2+\lambda\right)^{2}+\left(E-E_{R, k}(0)-\eta s\right)^{2}\right]\left[\left(\Gamma_{k} / 2\right)^{2}+\left(E-E_{R, k}(0)-\eta s\right)^{2}\right]}=0 .
$$

As in the case $N=1$, we observe that the l.h.s. of (11.22) for $\lambda=0$ is equal to the $s$-derivative of the l.h.s. of (11.14). Moreover, when $\lambda \rightarrow+\infty$, the l.h.s. of (11.22) converges to 1 , so if it is $<0$ for $\lambda=0$, it has to vanish for some $\lambda>0$. Hence, as in the case $N=1$, we get:

Proposition XI.1 Let $z$ be a fixed point of (11.18), so that $s=\|z\|^{2}$ solves (11.14). If the $s$-derivative of the l.h.s. of (11.14) is $<0$, then $z$ is not an attractive fixed point.

We now pass to the small- $\Gamma$ limit, where (11.14) is replaced by (11.15) and we keep the simplifying assumption (11.17).

Proposition XI.2 (small- $\Gamma$ limit) Assume that the intervals (11.10) are disjoint and let $z$ be a fixed point of (11.18). Then $z$ is attractive precisely when the s-derivative of the l.h.s. of (11.13) is $>0$.

Proof. The $s$-derivative of the l.h.s. of (11.15) is

$$
1-\sum_{j=0}^{N-1} \frac{2 \pi \eta\left(g\left|\mathcal{B}_{j}\right|^{2}\right)^{\prime}\left(E_{R, j}(0)+\eta s\right)}{\Gamma_{j}}
$$

where $\left(g\left|\mathcal{B}_{j}\right|^{2}\right)^{\prime}=\partial_{E}\left(g\left|\mathcal{B}_{j}\right|^{2}\right)$. On the other hand, in the small- $\Gamma$ limit, the equation (11.22) for the eigenvalues of the linearization becomes as in section VIII 


$$
\begin{aligned}
& 1-2 \eta \sum_{k ; \Gamma_{k} / 2+\operatorname{Re} \lambda>0} \frac{\pi\left(g\left|\mathcal{B}_{k}\right|^{2}\right)^{\prime}\left(E_{R, k}(0)+\eta s\right)}{\Gamma_{k}+\lambda} \\
& +2 \eta \sum_{k ; \Gamma_{k} / 2+\operatorname{Re} \lambda<0} \frac{\pi\left(g\left|\mathcal{B}_{k}\right|^{2}\right)^{\prime}\left(E_{R, k}(0)+\eta s\right)}{\lambda}=0 .
\end{aligned}
$$

We are only interested in the possible existence of solutions to this equation with $\operatorname{Re} \lambda \geq 0$, and for such $\lambda(11.24)$ reduces to

$$
1-2 \eta \sum_{k=0}^{N-1} \frac{\pi\left(g\left|\mathcal{B}_{k}\right|^{2}\right)^{\prime}\left(E_{R, k}(0)+\eta s\right)}{\Gamma_{k}+\lambda}=0
$$

If $\lambda$ is a solution, then by the condition that the intervals (11.16) are disjoint, only one term in the last sum, say for $k=m$, is $\neq 0$, so that (11.25) becomes

$$
1-2 \eta \frac{\pi\left(g\left|\mathcal{B}_{m}\right|^{2}\right)^{\prime}\left(E_{R, m}(0)+\eta s\right)}{\Gamma_{m}+\lambda}=0
$$

while the expression (11.23) becomes

$$
1-2 \eta \frac{\pi\left(g\left|\mathcal{B}_{m}\right|^{2}\right)^{\prime}\left(E_{R, m}(0)+\eta s\right)}{\Gamma_{m}} .
$$

It is then easy to see that the solution of (11.26) has a negative real part precisely when the expression (11.27) is positive, and this concludes the proof of the last proposition.

When the intervals (11.16) have non-empty intersections, the situation is more complicated, and the following example is an indication that the last proposition may be false.

Example. There exist $\Gamma_{1}, \Gamma_{2}>0, a_{1}, a_{2} \in \boldsymbol{R}$, such that $1-\left(a_{1} / \Gamma_{1}+a_{2} / \Gamma_{2}\right)>0$, while $1-\left(a_{1} /\left(\Gamma_{1}+\lambda\right)+\right.$ $\left.a_{2} /\left(\Gamma_{2}+\lambda\right)\right)=0$ for some positive $\lambda$. Indeed, choose $\Gamma_{1}=1, a_{1}=2, \Gamma_{2}=\delta>0$ very small, $a_{2}=-2 \delta$. Then $1-a_{1} / \Gamma_{1}-a_{2} / \Gamma_{2}=1>0$. If $\delta<<\lambda_{0}<<1$, we have

$$
1-\frac{a_{1}}{\Gamma_{1}+\lambda_{0}}-\frac{a_{2}}{\Gamma_{2}+\lambda_{0}} \approx-1
$$

Hence $1-\left(a_{1} /\left(\Gamma_{1}+\lambda\right)+a_{2} /\left(\Gamma_{2}+\lambda\right)\right)$ must vanish for some $\lambda$ between 0 and $\lambda_{0}$.

As in section $\mathbb{\amalg}$, we can derive a simplified differential equation for $\left(s_{0}(t), \ldots, s_{N-1}(t)\right)$, where $s_{j}(t)=\left\|z_{j}(t, \cdot)\right\|^{2}$, so that $s(t)=\sum_{j=0}^{N-1} s_{j}(t)$. We drop the simplifying assumption (11.17), but keep, for simplicity, the assumption that $\mathcal{B}_{j}$ are independent of $t$. Assume that $z(t, E)=\left(z_{0}(t, E), \ldots, z_{N-1}(t, E)\right)$ is a solution of (11.12) which has existed for a long time with a uniformly bounded norm. As in section IX, we take the scalar product of (11.12) with $z_{j}$ and get

$$
\frac{d}{d t} s_{j}(t)=-\Gamma_{j}(s)+2 \operatorname{Re}\left\langle z_{j} \mid \mathcal{B}_{j}\right\rangle
$$

Using

$$
z_{j}(t, E)=\int_{-\infty}^{t} d t^{\prime} e^{i\left(E-E_{R, j}(0)\right)\left(t-t^{\prime}\right)-\int_{t^{\prime}}^{t} d t^{\prime \prime} \Gamma_{j}\left(s\left(t^{\prime \prime}\right)\right) / 2-i \eta_{j} \int_{t^{\prime}}^{t} d t^{\prime \prime} s\left(t^{\prime \prime}\right)} \mathcal{B}_{j}\left(s\left(t^{\prime}\right), E\right)
$$

and, under the assumption that $s\left(t^{\prime}\right)$ is slowly varying, we get as in section $\mathrm{IX}$

$$
2 \operatorname{Re}\left\langle z_{j} \mid \mathcal{B}_{j}\right\rangle \simeq 2 \pi\left(g\left|\mathcal{B}_{j}\right|^{2}\right)\left(s(t), E_{R, j}(0)+\eta_{j} s(t)\right),
$$

and the simplified equations

$$
\frac{d}{d t} s_{j}(t)=-\Gamma_{j}(s(t))\left[s_{j}(t)-2 \pi \frac{\left(g\left|\mathcal{B}_{j}\right|^{2}\right)\left(s(t), E_{R, j}(0)+\eta_{j} s(t)\right)}{\Gamma_{j}(s(t))}\right],
$$


for $j=0,1, \ldots, N-1$, and $s=\sum_{j=0}^{N-1} s_{j}$. We notice that the region defined by $s_{j} \geq 0$ for $0 \leq j \leq N-1$ is stable under the forward flow associated to the system (11.31). Moreover, if $\left(s_{0}, \ldots, s_{N-1}\right)$ is a fixed point of this system, then we get precisely (11.15). Conversely, if $s$ is a solution of (11.15), then

$$
s_{j}=2 \pi \frac{\left(g\left|\mathcal{B}_{j}\right|^{2}\right)\left(s, E_{R, j}(0)+\eta_{j} s\right)}{\Gamma_{j}(s)}
$$

defines the corresponding unique fixed point solution with $s=\sum_{j=0}^{N-1} s_{j}$.

We end this section by investigating the linearization of (11.31) at a fixed point solution, under the simplifying assumption (11.17). An easy calculation shows that the linearization is given by

$$
\mathcal{M}\left(\begin{array}{c}
v_{0} \\
\vdots \\
v_{N-1}
\end{array}\right)=\left(\begin{array}{c}
-\Gamma_{0} v_{0}+2 \pi \eta s\left(g\left|\mathcal{B}_{0}\right|^{2}\right)^{\prime}\left(E_{R, 0}(0)+\eta s\right) \sum_{k=0}^{N-1} v_{k} \\
\vdots \\
-\Gamma_{N-1} v_{N-1}+2 \pi \eta s\left(g\left|\mathcal{B}_{N-1}\right|^{2}\right)^{\prime}\left(E_{R, N-1}(0)+\eta s\right) \sum_{k=0}^{N-1} v_{k}
\end{array}\right)
$$

If $\lambda$ is an eigenvalue of $\mathcal{M}$ with $\Gamma_{j}+\lambda \neq 0$ for every $j$, and ${ }^{t}\left(v_{0}, \ldots, v_{N-1}\right)$ a corresponding non-trivial eigenvector, we have

$$
v_{j}=2 \pi \eta \frac{\left(g\left(\left|\mathcal{B}_{j}\right|^{2}\right)^{\prime}\left(E_{R, j}(0)+\eta s\right)\right.}{\Gamma_{j}+\lambda} \sum_{k=0}^{N-1} v_{k}
$$

Then necessarily the sum is $\neq 0$, and by summing these $N$ relations, we see that $\lambda$ is an eigenvalue precisely when (11.25) holds. We finally get:

Proposition XI.3 Under the simplifying assumption (11.17) and in the small-Г limit, let $z$ be a fixed point of (11.18) and let $\left(s_{0}, \ldots, s_{N-1}\right)$ be the corresponding fixed point solution of (11.31). Then the linearizations of (11.18) and (11.31) at the corresponding fixed points have the same eigenvalues in the right half plane Red $\geq 0$ (given by (11.25)). In particular, $z$ is an attractive fixed point for (11.18) precisely when $\left(s_{0}, \ldots, s_{N-1}\right)$ is an attractive fixed point for (11.31).

\section{ACKNOWLEDGMENTS}

We thank G. Jona-Lasinio for interesting discussions on the conceptual aspects of the physical model. We also thank G. Perelman for interesting discussions on the mathematical aspects and acknowledge that she independently found the basic differential equation (9.7). Partial support of INFN, Iniziativa Specifica RM6, is acknowledged.

$\dagger \quad$ presilla@roma1.infn.it

¥ johannes@orphee.polytechnique.fr

[1] For a general discussion of heterostructures see F. Capasso and S. Datta, Phys. Today 43, No. 2, 74 (1990).

[2] Mesoscopic phenomena in solids, edited by B. L. Altshuler, P. A. Lee, and R. A. Webb (Elsevier, New York, 1991).

[3] G. Timp, Ballistic transport in one dimension, in Ref. [2], p. 273.

[4] The crystals which compose the heterostructure may have different periodic potentials giving rise to a layer-dependent effective mass as discussed, for example, by G. T. Einevoll, P. C. Hemmer, and J. Thomsen, Phys. Rev. B 42, 3485 (1990). Here, we will suppose to have a constant effective mass which is a reasonable assumption for GaAs-AlGaAs heterostructures.

[5] D. Pines and P. Nozières, The Theory of Quantum Liquids (W. A. Benjamin, New York, 1966).

[6] B. Ricco and M. Ya. Azbel, Phys. Rev. B 29, 1970 (1984).

[7] C. Presilla, G. Jona-Lasinio, and F. Capasso, Phys. Rev. B 43, 5200 (1991).

[8] B. A. Malomed and M. Ya. Azbel, Phys. Rev. B 47, 10402 (1993).

[9] G. Jona-Lasinio, C. Presilla, and F. Capasso, Phys. Rev. Lett. 68, 2269 (1992).

[10] T. Sajoto, J. J. O'Shea, S. Bhargava, D. Leonard, M. A. Chin, and V. Narayanamurti, Phys. Rev. Lett. 74, 3427 (1995).

[11] V. J. Goldman, D. C. Tsui, and J. E. Cunningham, Phys. Rev. Lett. 58, 1256 (1987). 
[12] F. W. Sheard and G. A. Toombs, Appl. Phys. Lett. 52, 1228 (1988); Semicond. Sci. Technol. 7, B460 (1992).

[13] A. N. Korotkov, D. V. Averin, and K. K. Likharev, Physica B 165 \& 166, 927 (1990).

[14] K. L. Jensen and F. A. Buot, Phys. Rev. Lett. 66, 1078 (1991).

[15] Y. Abe, Semicond. Sci. Technol. 7, B498 (1992).

[16] C. Presilla and J. Sjöstrand, Hysteresis and ghost stationary currents in biased resonant tunneling heterostructures, e-print archive cond-mat/9602047.

[17] G. Jona-Lasinio, C. Presilla, and J. Sjöstrand, Ann. Phys. 240, 1 (1995).

[18] A. Zaslavsky, V. J. Goldman, D. C. Tsui, and J. E. Cunningham, Appl. Phys. Lett. 53, 1408 (1988).

[19] R. Bonifacio and P. Meystre, Optics Comm. 29, 131 (1979); G. Broggi, L. A. Lugiato, and A. Colombo, Phys. Rev. A 32, 2803 (1985).

[20] J. Kastrup, H. T. Grahn, K. Ploog, F. Prengel, A. Wacker, and E. Schöll, Appl. Phys. Lett. 65, 1808 (1993).

[21] N. G. Sun and G. P. Tsironis, Phys. Rev. B 51, 11221 (1995).

[22] A. Wacker and E. Schöll, J. Appl. Phys. 78, 7352 (1995).

[23] J. Aguilar and J. M. Combes, Comm. Math. Phys. 22, 269 (1971). See also P. Lochak, Ann. Inst. Henri Poincaré A 39, 119 (1983) and W. Hunziker, Ann. Inst. Henri Poincaré A 45, 339 (1986).

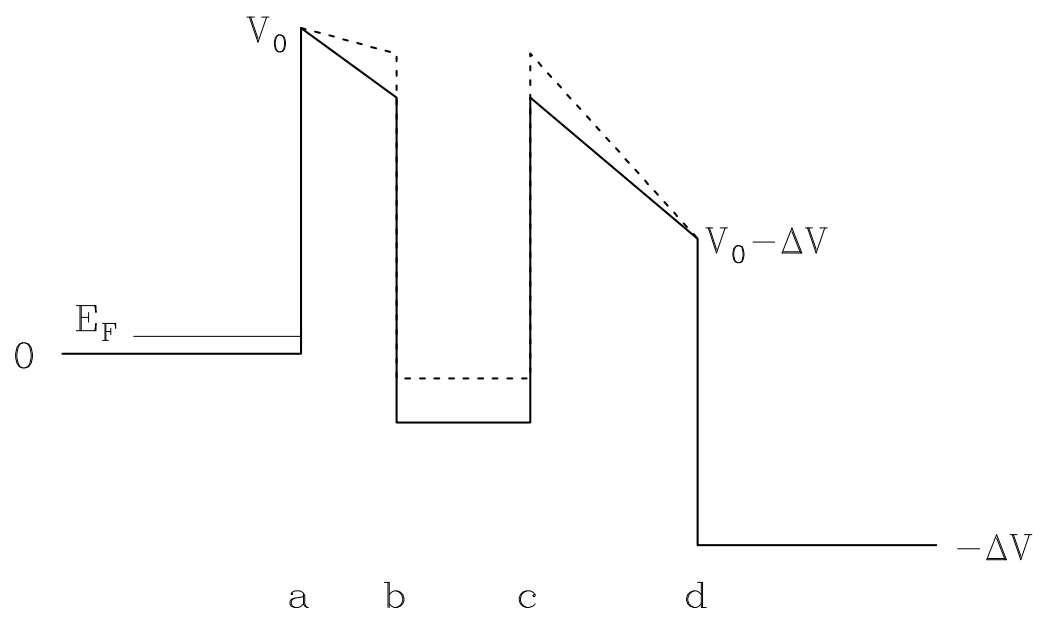

FIG. 1. Potential $V(x)$ representing the band profile modified by the external bias energy $\Delta V$ (solid line) and total potential $V(x)+W(s, x)$ including the electrostatic contribution due to electrons trapped in the well with sheet density $s$ (dashed line). 

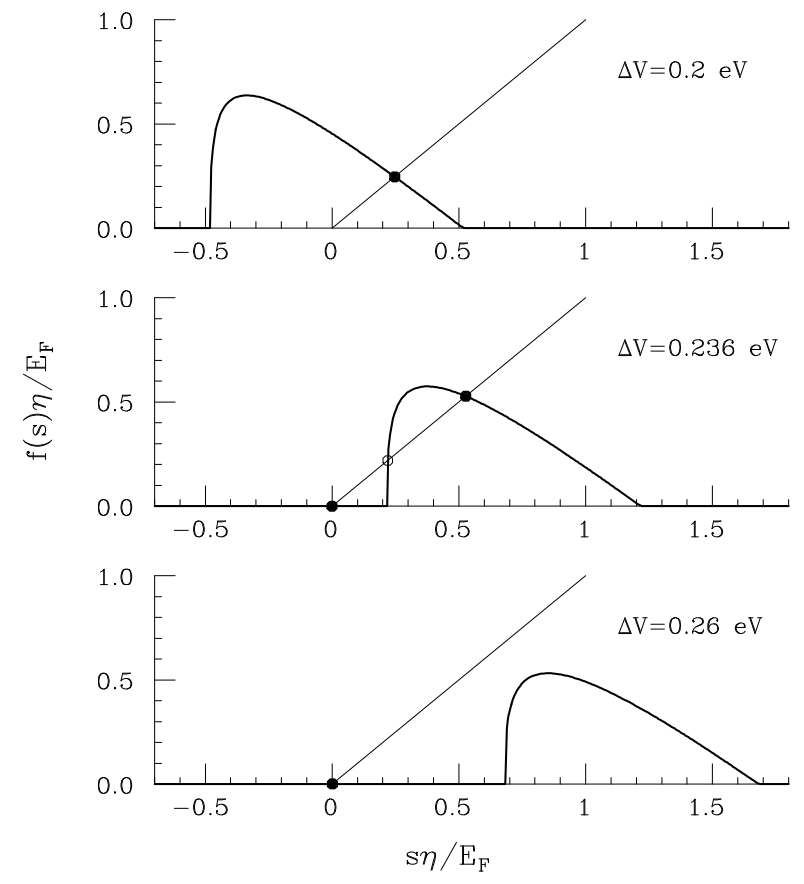

FIG. 2. Graphical solution of the equation $s=f(s)$ for different values of the bias energy $\Delta V$. Note that the support of $f(s)$ has width $\Delta s \simeq E_{F} / \eta$ (equality strictly holds at zero temperature). The example shown here corresponds to a typical GaAs-AlGaAs heterostructure in which the parameters described in the text have the following values: $n_{D}=2 \times 10^{17} \mathrm{~cm}^{-3}$, $T=1 \mathrm{~K}, b-a=40 \AA, c-b=56 \AA, d-c=70 \AA, V_{0}=0.34 \mathrm{eV}, \varepsilon=11.44$, and $m^{*}=0.067 m$, where $m$ is the free electron mass.

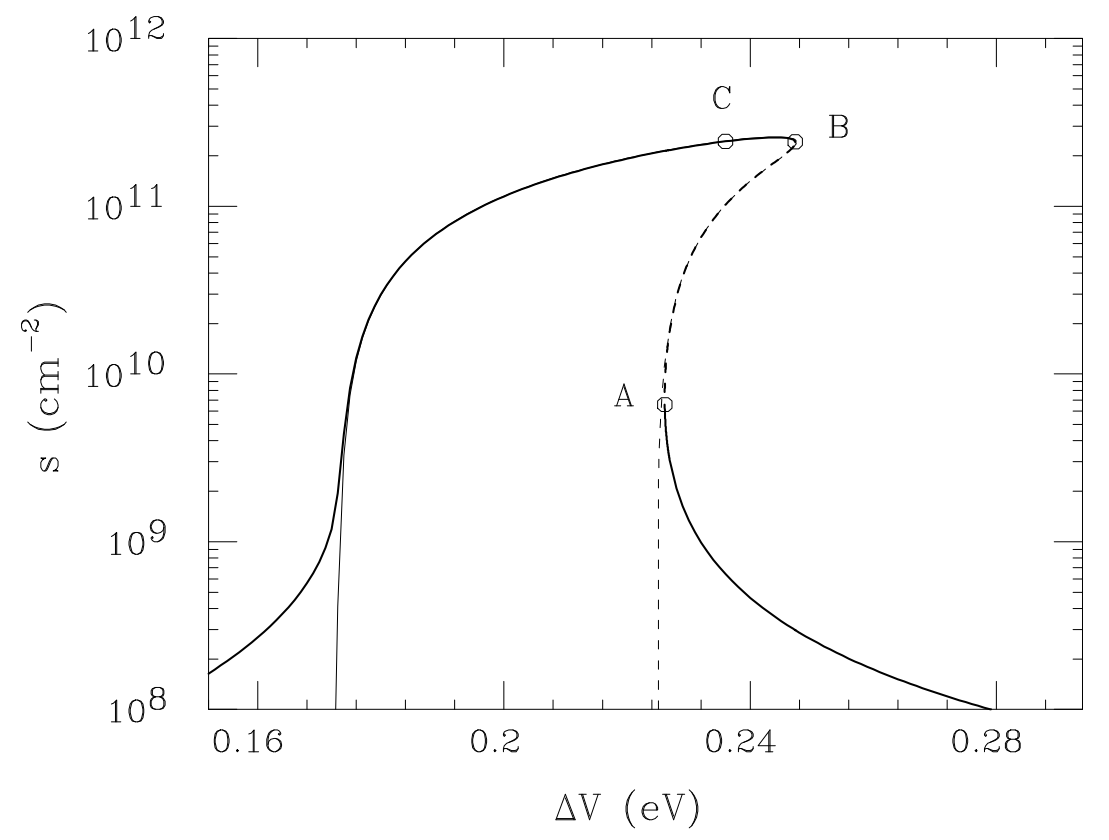

FIG. 3. Fixed point solutions of the sheet density of electrons in the well $s$ as a function of the bias energy $\Delta V$ in the same case of Fig. 2. The thick line is the exact case (7.4) and the thin line the small- $\Gamma$ approximation (8.1). Unstable solutions are represented by dashed lines (both thick and thin). 


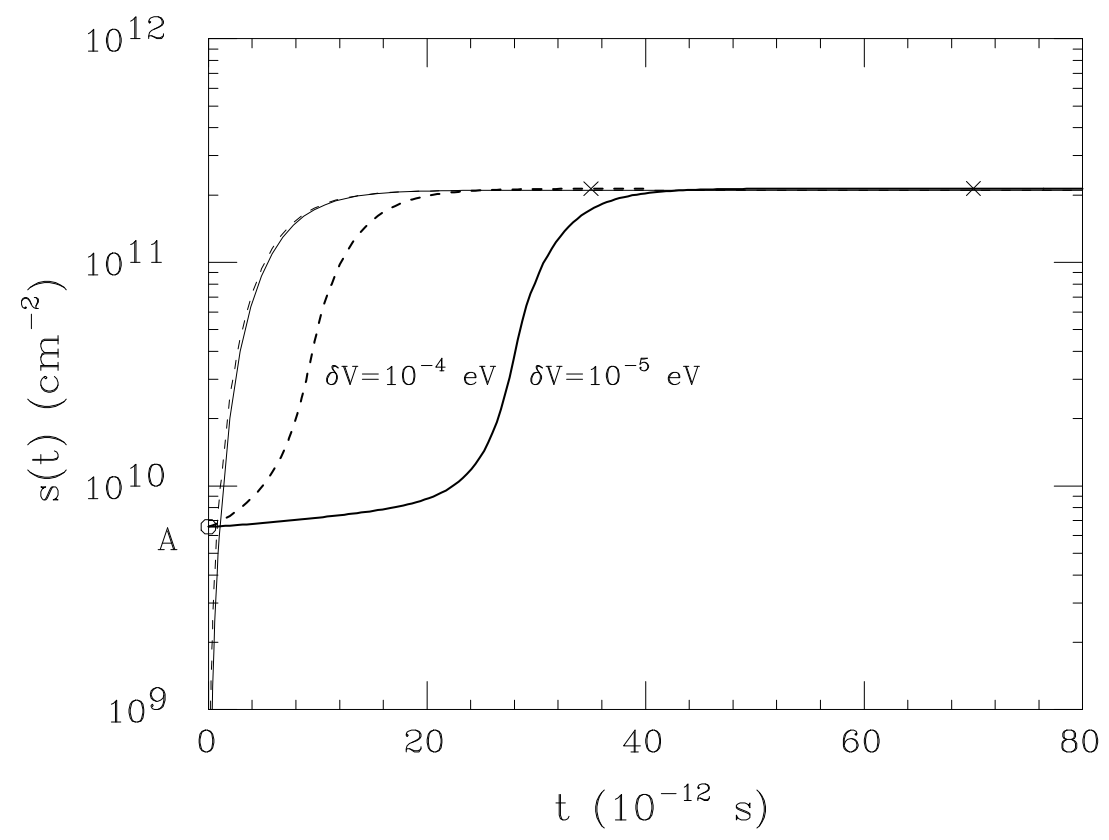

FIG. 4. Sheet density of electrons in the well $s(t)$ as a function of time after an instantaneous decrease $\delta V$ of the bias energy from the point $A$ of Fig. 3 (thick lines). The crosses are the fixed point solutions at bias $\Delta V_{A}-\delta V$ where $s(t)$ is expected to converge. The thin lines are the corresponding small- $\Gamma$ approximation starting from $s(0)=0$.

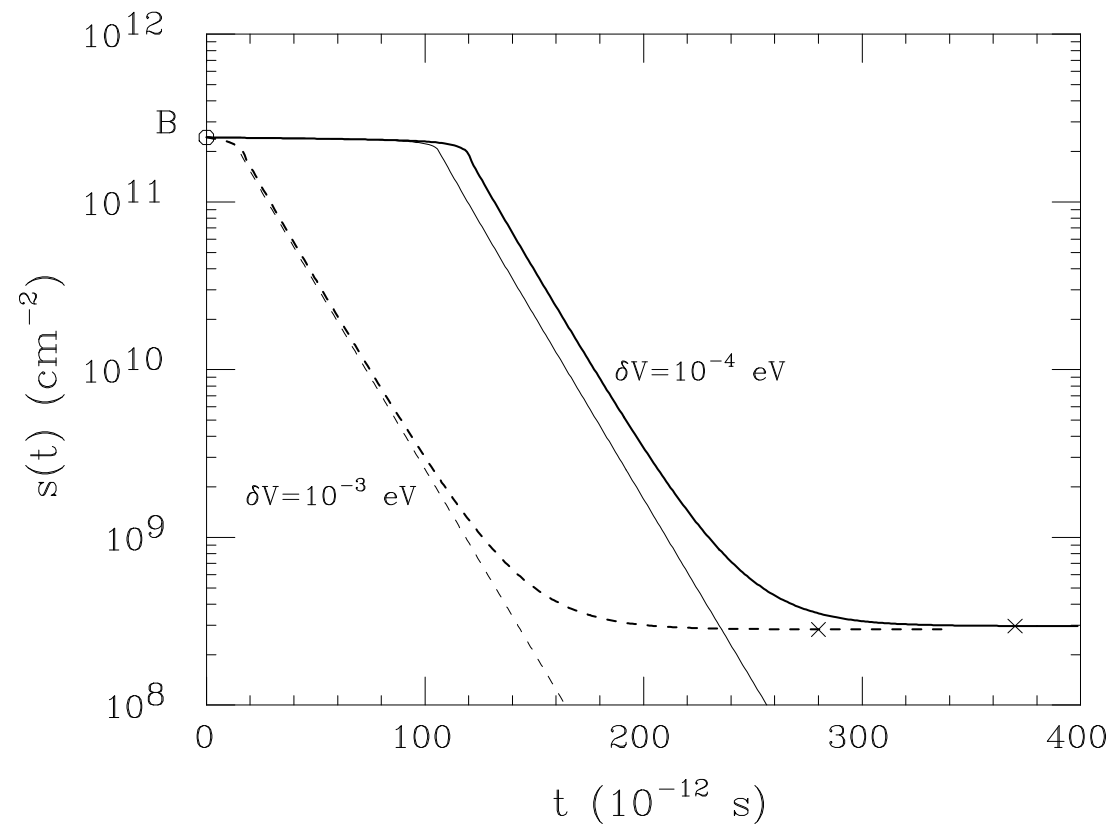

FIG. 5. Sheet density of electrons in the well $s(t)$ as a function of time after an instantaneous increase $\delta V$ of the bias energy from the point $B$ of Fig. 3 (thick lines). The crosses are the fixed point solutions at bias $\Delta V_{B}+\delta V$ where $s(t)$ is expected to converge. The thin lines are the corresponding small- $\Gamma$ approximation. For $\delta V$ not too large a ghost fixed-point solution is observed with $s(t)$ decaying linearly for $t \leq t_{\mathrm{g}}$ and $t_{\mathrm{g}}$ defined by the condition $E_{R}\left(s\left(t_{g}\right)\right)=0$. 


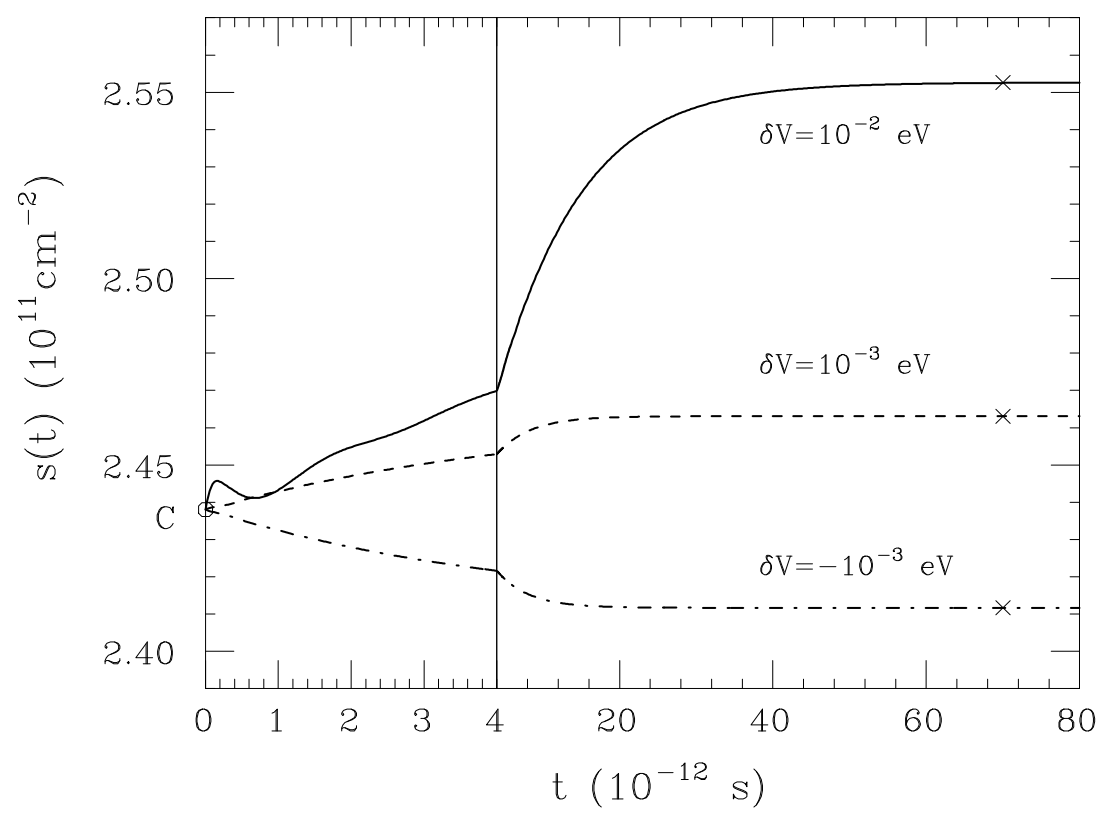

FIG. 6. Sheet density of electrons in the well $s(t)$ as a function of time after an instantaneous change $\delta V$ of the bias energy from the point $C$ of Fig. 3. The crosses are the fixed point solutions at bias $\Delta V_{C}+\delta V$ where $s(t)$ is expected to converge. For $|\delta V|$ not too small damped oscillations are seen at the ps scale.
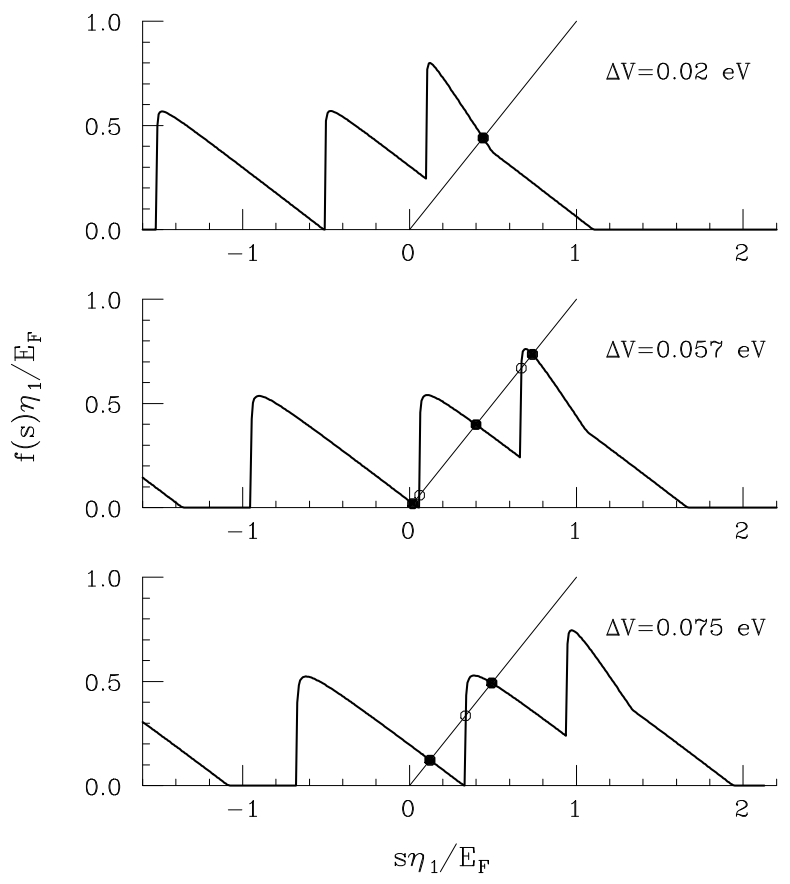

FIG. 7. Graphical solution of the equation $s=f(s)$ for different values of the bias energy $\Delta V$ in a multiple-resonance case. We used the same parameters of Fig. 2 except $b-a=20 \AA, c-b=360 \AA, d-c=50 \AA$. 


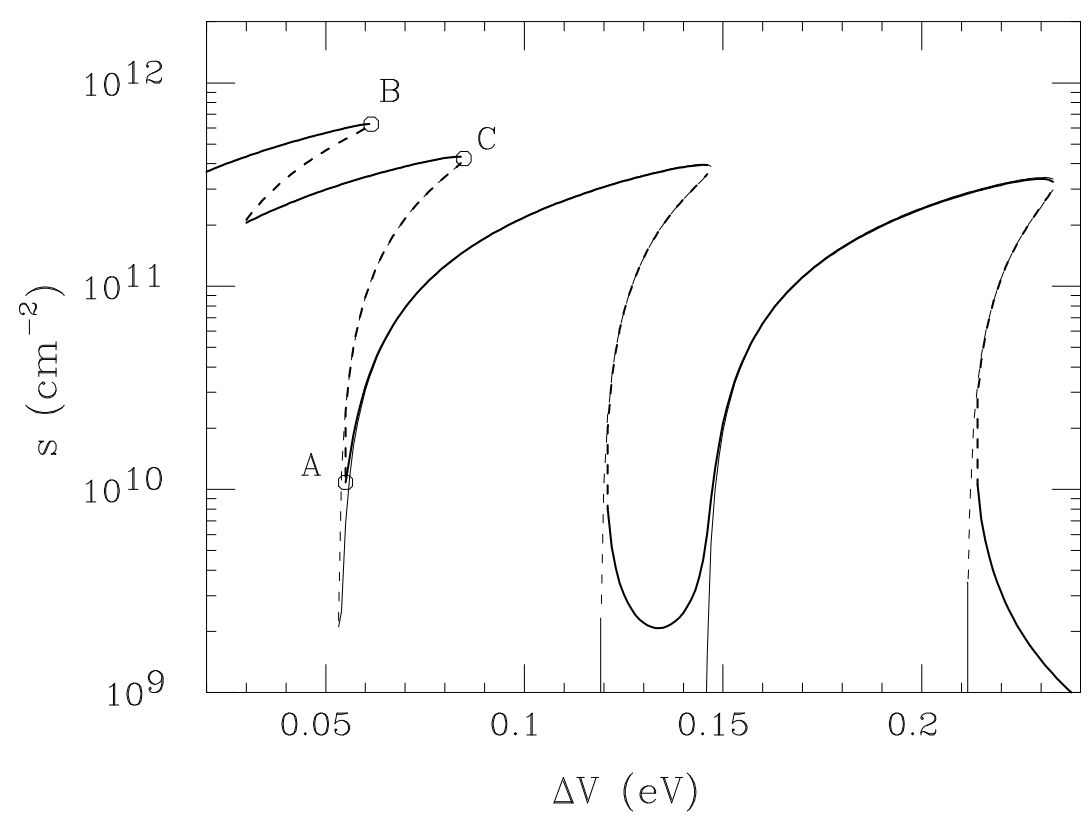

FIG. 8. Fixed point solutions of the sheet density of electrons in the well $s$ as a function of the bias energy $\Delta V$ in the same case of Fig. 7. The thick line is the solution of Eq. (11.14) and the thin line the small- $\Gamma$ limit (11.15). Possibly unstable solutions are represented by dashed lines (both thick and thin).

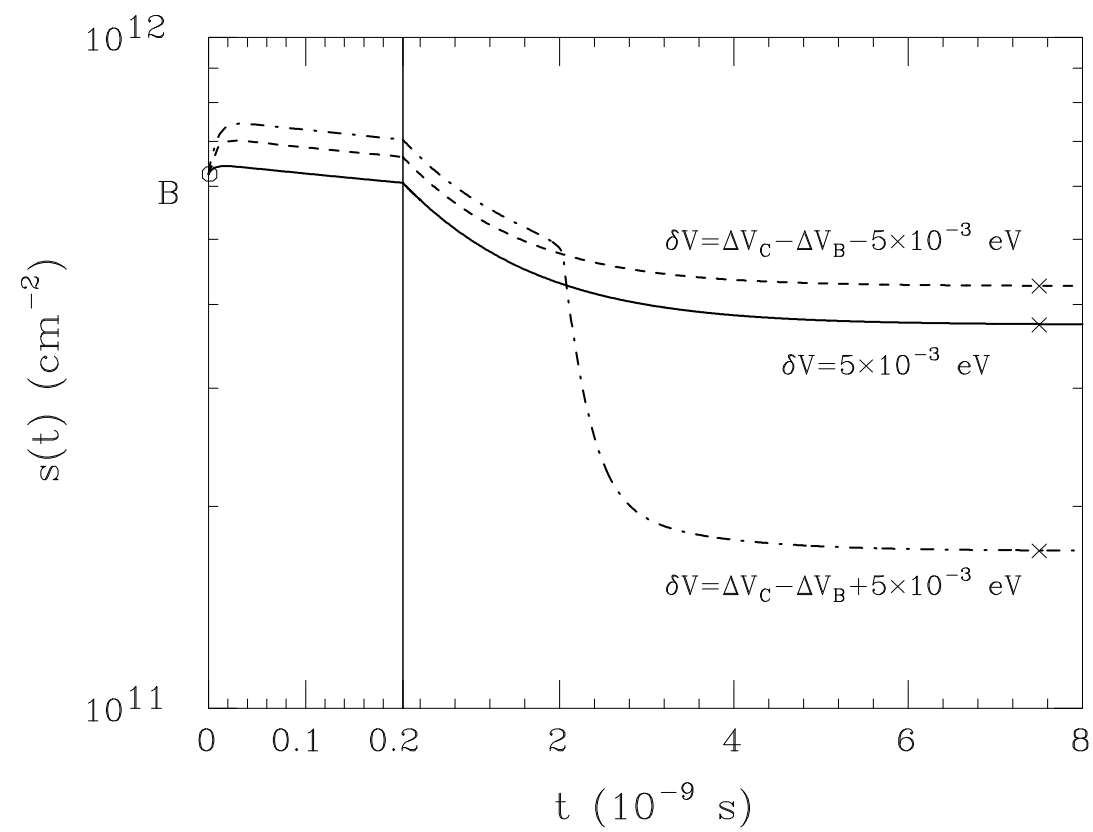

FIG. 9. Sheet density of electrons in the well $s(t)$ as a function of time after an instantaneous increase $\delta V$ of the bias energy from the point $B$ of Fig. 8. The crosses are the fixed point solutions at bias $\Delta V_{B}+\delta V$ where $s(t)$ is expected to converge. 